\title{
Theory of Light Emission in Sonoluminescence as Thermal Radiation
}

\author{
Wang-Kong Tse* and P.T. Leung ${ }^{\dagger}$ \\ Physics Department and Institute of Theoretical Physics, \\ The Chinese University of Hong Kong, Shatin, Hong Kong SAR, China
}

(Dated: November 4, 2018)

\begin{abstract}
Based on the model proposed by Hilgenfeldt at al. [Nature 398, 401 (1999)], we present here a comprehensive theory of thermal radiation in single-bubble sonoluminescence (SBSL). We first invoke the generalized Kirchhoff's law to obtain the thermal emissivity from the absorption crosssection of a multilayered sphere (MLS). A sonoluminescing bubble, whose internal structure is determined from hydrodynamic simulations, is then modelled as a MLS and in turn the thermal radiation is evaluated. Numerical results obtained from simulations for argon bubbles show that our theory successfully captures the major features observed in SBSL experiments.

PACS numbers: 78.60.Mq, 42.25.Bs, 52.25.Os, 52.40.Db
\end{abstract}

\section{INTRODUCTION}

Single-bubble sonoluminescence (SBSL or simply SL), first discovered in 1989, is a phenomenon of periodic light emission by an oscillating gas bubble trapped in the pressure antinode of a standing ultrasound wave in water (or other fluids) (see 1, 2] for detailed reviews on SBSL). The oscillating bubble is stable enough to survive many days through billions of acoustic cycles while the produced flashes are highly regular and incandescent. The width of the emitted light pulse is around $10-100 \mathrm{ps}$ with a peak power of the order $10 \mathrm{~mW}$ [1, 2, 3, 4]. The light pulse has nearly a gaussian shape with a slight asymmetry, which is basically identical in the red and UV portions of the spectrum [4], and Hiller et al. [5] further confirmed that the pulse width and the emission time were independent of wavelength. However, in an interesting twist, Moran et al. [6] demonstrated that the pulse width did exhibit a mild dependence on wavelength at $3{ }^{\circ} \mathrm{C}$, but not at $24^{\circ} \mathrm{C}$. Besides, the power spectrum of the emitted light was found to be broadband without any characteristic line, decreasing from the UV portion towards the red in a way that bore a resemblance to a blackbody spectrum [5, 7, 8].

SBSL has become an intriguing topic and an arena for experimentalists and theorists alike since its discovery. Numerous attempts have been made to study the bubble motion using classical bubble dynamics and sophisticated computational fluid mechanics (CFM) 9, 10, 11, 12, 13, 14, 15, 16. It is generally believed that the bubble is heated to temperatures of tens of thousands and shock waves and plasma could be generated during the contraction of the bubble. Various proposals have been put forward to explain the light emission mechanism, including surface blackbody radiation [1, 17, 17, 18], neutral

\footnotetext{
*Present address: Condensed Matter Theory Center, Department of Physics, University of Maryland at College Park, College Park, Maryland 20742-4111, USA

†email: ptleung@phy.cuhk.edu.hk
}

and ion bremsstrahlung 12, 13, 16, 19, 20, 21, collisioninduced emission [22, 23], quantum vacuum radiation [24], confined-electron model 25], proton-tunnelling [26], and nuclear fusion 27, 28, 29. Some of these proposals, e.g. [1, 7, 12, 13, 16, 17, 18, 19, 20, 21, 22, 23], attributed light emission in SBSL to the high temperature attained in the bubble and are classified as thermal radiation schemes in this paper. While qualitatively reproducing the spectra detected in SBSL, most of such thermal schemes failed to explain why the pulse width is wavelength-independent as measured in some experiments [4, 5]. Owing to this major drawback of thermal schemes, researchers were forced to look into other non-thermal and more exotic models (see, e.g. [24, 25, 26, 27, 28, 29]).

To reconcile the success and the drawback of thermal radiation schemes, Hilgenfeldt, Grossmann and Lohse 20, 21 took the finite opacity of the bubble into consideration and obtained a wavelength-independent pulse width for argon bubbles. The impact of Hilgenfeldt et al.'s work is huge and, to some extent, resurrects the thermal radiation scheme. However, it is worthwhile to note that the approach adopted in 20, 21] is deemed a simplified version of the emission mechanism of SBSL as several physical processes have not been included in the proposal [30]. For example, the sonoluminescing bubble is modelled as a uniform one and the fluid dynamics inside the bubble has been neglected from the outset [20, 21]. Besides, the Kirchhoff's law used in [20, 21] to evaluate thermal emissivity of the bubble has completely ignored the wave nature of light.

In addition, existing literature (e.g. 2, 31, 32, 33]) demonstrates there exists a gap in the theoretical treatment of light emission mechanism of sonoluminescence, in that blackbody radiation and thermal bremsstrahlung are often ascribed as separate possible mechanisms of SBSL. Often, Planck's formula for blackbody radiation 31, 32 and absorption coefficients for thermal bremsstrahlung in vacuum [20,21] are applied separately in these cases, and the question of whether the bubble is opaque enough to demonstrate blackbody radiation is argued in a rather hand-waving manner by compar- 
ing the photon mean path with an estimated size of the light-emitting region. In our view, this is because of the lack of a single theory which can take account of both mechanisms in a finite-sized environment (i.e. the bubble) in a unifying manner. In a recent experimental paper 33], Flannigan et al. demonstrated conclusively the existence of a plasma state inside the bubble, and hence thermal bremsstrahlung is an inevitable consequence because of the motion of the electrons and ions. We emphasize that blackbody radiation and thermal bremsstrahlung are nothing but one single emission mechanism manifested upon the degree of optical thickness of the bubble; and to this purpose, in this paper we have developed a coherent theory unifying both aspects in the context of SBSL. Within the framework of this theory, when the bubble becomes optically thick enough, the thermal bremsstrahlung manifest itself asymptotically as blackbody radiation.

First, we will consider thermal emission in a realistic sonoluminescing bubble that is non-uniform in temperature as well as density. To properly describe processes of thermal emission and absorption in a finite volume with a size comparable to the wavelength of light in a consistent manner, the generalized Kirchhoff's law is used in our paper [34]. Second, state- of-the-art CFM is applied here to evaluate the temperature and density distributions in the bubble 14, 15]. Through such elaboration of the thermal radiation scheme [20, 21], we succeed in obtaining an emission spectrum that agrees nicely with the experimental data as summarized in [1] and, in addition, resolve the dilemma of whether the pulse width is dependent on the wavelength.

The rest of this paper is organized as follows. We first present the generalized Kirchhoff's law in Sec. III and show in Sec. III that it leads to the formula for emissivity used in 20, 21] in certain limits. In Secs. IV and V we make use of the generalized Kirchhoff's law to derive the spectral radiance of a heated multilayered sphere. In Secs. VI and VII respectively we furnish the plasma model and CFM used in the present paper. We then present relevant numerical results in Sec. VIII and conclude our paper in Sec. IX

\section{THERMAL RADIATION}

In SL, the high temperature reached inside the bubble ionizes the gas content, forming a partially ionized plasma [16, 20, 21, 35, 36]. Besides having finite optical thickness, the bubble has a size $\sim 0.1-1 \mu \mathrm{m}$ near the instant of maximum compression, which is comparable to the wavelength of the emitted light ranging from $200 \mathrm{~nm}$ to $800 \mathrm{~nm}$. Hence, the assumption of geometric optics is invalid. To properly take account of the effects of finite absorption, wave reflection and diffraction, we employ in this paper the generalized Kirchhoff's law 34], which yields the 'classical' form of Kirchhoff's law as an asymptotic limit, to compute the power spectrum.
We first review the statement of the 'classical' form of Kirchhoff's law (see, e.g. 37]). Consider an isotropic absorbing medium with complex dielectric constant $\epsilon=$ $\epsilon_{R}+i \epsilon_{I}$, refractive index $n=\sqrt{\epsilon}=n_{R}+i n_{I}$, and a typical size $d$. If the medium is in thermal equilibrium at temperature $T$ and $d$ is large compared with the wavelength of light $\lambda$ so that the geometric optics approximation holds, then the classical form of Kirchhoff's law relates the emission coefficient $\eta(\omega)$ and the absorption coefficient $\kappa(\omega) \equiv 2 n_{I} \omega / c$ at (angular) frequency $\omega \equiv 2 \pi f$ as 37]:

$$
\eta / \kappa=n_{R}^{2} B_{\omega}(T)
$$

where

$$
B_{\omega}(T)=\frac{\hbar \omega^{3}}{8 \pi^{3} c^{2}\left(e^{\hbar \omega / k_{B} T}-1\right)}
$$

is the (frequency) spectral light intensity of a blackbody for one polarization, with $c$, $\hbar$ and $k_{B}$ being the speed of light in vacuum, the Planck constant $h$ divided by $2 \pi$, and the Boltzmann constant, respectively. We remark that, besides the assumption $\lambda \ll d$, its application is justified only to a volume emitter which is optically thin.

The generalized Kirchhoff's law [34] is a generalization of the classical form of Kirchhoff's law to all sizes $d$ and optical thickness of a finite-size emitter, and can be derived from the Maxwell equations:

$$
\begin{aligned}
& \nabla \cdot \boldsymbol{D}(\boldsymbol{r}, t)=\rho_{n}(\boldsymbol{r}, t), \\
& \nabla \cdot \boldsymbol{B}(\boldsymbol{r}, t)=0, \\
& \nabla \times \boldsymbol{E}(\boldsymbol{r}, t)+\frac{\partial \boldsymbol{B}(\boldsymbol{r}, t)}{\partial t}=0, \\
& \nabla \times \boldsymbol{H}(\boldsymbol{r}, t)-\frac{\partial \boldsymbol{D}(\boldsymbol{r}, t)}{\partial t}=\boldsymbol{J}_{n}(\boldsymbol{r}, t) .
\end{aligned}
$$

Here the electric field $\boldsymbol{E}$, the magnetic induction $\boldsymbol{B}$, the displacement field $\boldsymbol{D}$ and the magnetic field strength $\boldsymbol{H}$ are generated by the fluctuating charge density $\rho_{n}$ and current density $\boldsymbol{J}_{n}$, which are direct consequence of the random thermal motions of the charges and satisfy the fluctuation-dissipation theorem (see, e.g. 38]):

$$
\begin{aligned}
& \left\langle\tilde{J}_{n, i}(\boldsymbol{r}, \omega) \tilde{J}_{n, j}^{*}\left(\boldsymbol{r}^{\prime}, \omega\right)\right\rangle \\
= & \frac{4 \omega}{\pi} \theta(\omega, T) \epsilon_{0} \epsilon_{I}(\boldsymbol{r}, \omega) \delta\left(\boldsymbol{r}-\boldsymbol{r}^{\prime}\right) \delta_{i j} .
\end{aligned}
$$

Here $\tilde{J}_{n, i}(\boldsymbol{r}, \omega)$ is the $i$-component of the Fourier transform of $\boldsymbol{J}_{n}(\boldsymbol{r}, t)$,

$$
\theta(\omega, T)=\frac{\hbar \omega}{2} \operatorname{coth} \frac{\hbar \omega}{2 k_{B} T}
$$

is the average energy of a quantum harmonic oscillator at temperature $T$, and $\epsilon_{0}$ is permittivity constant of vacuum.

In the presence of the thermal fluctuations $\rho_{n}$ and $\boldsymbol{J}_{n}$, all fields $\boldsymbol{E}, \boldsymbol{D}, \boldsymbol{H}$ and $\boldsymbol{B}$ are thermally fluctuating 
quantities. The set of stochastic equations, (2.3)-(2.6), together with the fluctuation-dissipation theorem, completely determine the statistics of the electromagnetic field of such a system and the relevant theory is termed the thermal electromagnetic theory (TET) 34].

The generalized Kirchhoff's law derived from TET [34] states that the spectral radiance $P_{\lambda}$, defined as the power emitted per unit wavelength interval and unit solid angle, is given by the following formula 34]:

$$
P_{\lambda}(\mathbf{n})=B_{\lambda}(T) \sigma_{\mathrm{abs}}(\mathbf{n}) .
$$

Here $P_{\lambda}(\mathbf{n})$ is the spectral radiance in the direction of $\mathbf{n} \equiv \mathbf{r} /|\mathbf{r}|, \sigma_{\text {abs }}(\mathbf{n})$ is the absorption cross-sectional area of the emitter for an electromagnetic wave illuminating the emitter from the direction of $\mathbf{n}$, and

$$
B_{\lambda}(T)=\frac{h c^{2}}{\lambda^{5}\left(e^{h c / \lambda k_{B} T}-1\right)}
$$

is the spectral light intensity in each polarization for an ideal blackbody. In (2.9) it is understood that $\sigma_{\text {abs }}$ is the sum of the absorption cross-sectional areas for incident light waves with two perpendicular polarizations. It is evident that $\sigma_{\mathrm{abs}}$ is equal to the power $P_{a}$ dissipated in the emitter for an incident plane wave carrying unit energy flux, which can be obtained from the following volume integral over the emitter:

$$
P_{a}=\frac{\omega}{2} \int \mathrm{d}^{3} r \epsilon_{0} \epsilon_{I}(\boldsymbol{r}, \omega)|\boldsymbol{E}(\boldsymbol{r}, \omega)|^{2} .
$$

It is worthwhile to note that $\boldsymbol{E}(\boldsymbol{r}, \omega)$ the electric field developed inside the emitter might be strongly enhanced at certain frequencies due to resonance effects and thus carries non-trivial frequency dependence [36].

Besides the spectral radiance, the integrated power of the emitted light pulse is another quantity measured in SBSL experiments and is simply the integral of Eq. (2.9) over the wavelength,

$$
P=\int_{\lambda_{1}}^{\lambda_{2}} \mathrm{~d} \lambda P_{\lambda}
$$

\section{GEOMETRIC OPTICS MODEL}

We now apply the generalized Kirchhoff's law summarized above to consider light emission in the uniform bubble model (UBM) as proposed in 20, 21]. We will see that the formula for the spectral radiance used in 20, 21] is only an approximate one that is valid only under certain restrictions.

To use a simple situation to elucidate the generalized Kirchhoff's law, we first calculate the power emitted from a slab. Consider a weakly absorbing slab illuminated normally by a plane-polarized plane wave with unit flux. Under the assumption that the slab (with thickness $L$ and

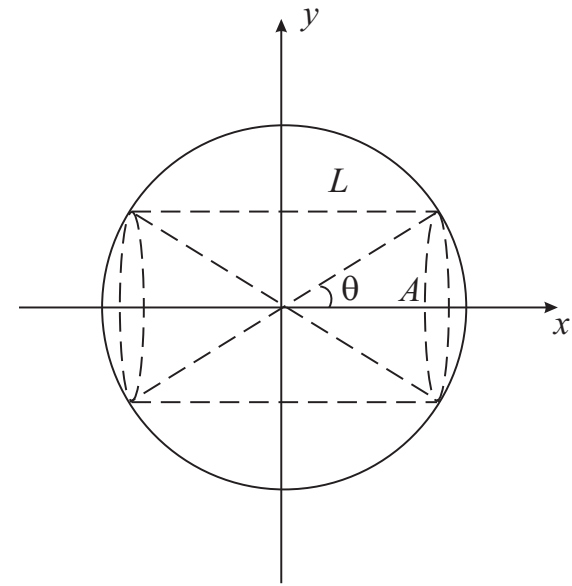

FIG. 1: The configuration of a uniform absorbing sphere.

area $A$ ) has dimensions sufficiently large so that multiple internal reflections can be neglected and the internal electric field can be represented by a single decaying, travelling wave only, the internal power loss can be found from Eq. (2.11):

$$
P_{a}=\frac{\omega \epsilon_{0} \epsilon_{I}}{\kappa c}\left(1-p_{\mathrm{ref}}\right) A\left(1-e^{-\kappa L}\right),
$$

Here $p_{\text {ref }}$ is the fraction of power reflected from the slab surface. For small absorption, the imaginary part of the refractive index $n_{I} \simeq \epsilon_{I} / 2$, so

$$
P_{\lambda}=2\left(1-p_{\text {ref }}\right) B_{\lambda}(T)\left(1-e^{-\kappa L}\right) A,
$$

where the factor of 2 properly takes care of the two possible polarizations. This result is consistent with that obtained from the standard radiative transfer theory $37,[39]$, upon which the spectral radiance obtained in 20,21$]$ is founded.

In the following we explicitly derive the formula for the spectral radiance that was used by Hilgenfeldt et al. in [20, 21] from Eq. (3.2). Fig. 1 shows a uniform sphere of radius $R$, which is divided into multiple thin cylindrical shells. Each of these shells, indicated by the dashed lines, subtends an angle of $\pi-2 \theta$ at the center, and has a length $L(\theta)=2 R \cos \theta$ along the $x$-direction and a differential cross-sectional area $d A=2 \pi R^{2} \sin \theta \cos \theta d \theta$. With the assumptions that (i) $p_{\text {ref }}=0$, (ii) effects of refraction and diffraction at the spherical interface are negligible, (iii) internal reflection is ignorable, and (iv) each of these shells can be considered as a slab with area $d A$ and thickness $L(\theta)$, the radiance per unit solid angle follows directly from Eq. (3.2) is given by:

$$
\begin{aligned}
P_{\lambda} & =\int \mathrm{d} A 2 B_{\lambda}(T)\left(1-e^{-\kappa z}\right) \\
& =2 \pi R^{2} B_{\lambda}(T)\left[1+\frac{e^{-2 \kappa R}}{\kappa R}+\frac{e^{-2 \kappa R}-1}{2 \kappa^{2} R^{2}}\right] .
\end{aligned}
$$

Multiplying this by the total solid angle $4 \pi$ straightforwardly yields the formula Eq. (17) in Ref. [20]. As seen 


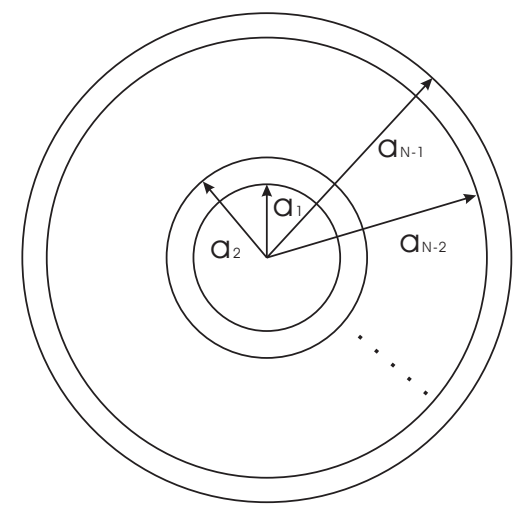

FIG. 2: An $N$-layered spherical system. The inner $N-1$ layers (the bubble) are absorbing, while the outermost layer (the surrounding fluid) is transparent.

from the derivation here, this formula is only valid under the assumptions mentioned above, through which the wave nature of light has been completely neglected. Such an emission model will be referred to as the geometric optics model (GOM) in the following discussion. As we will show in our numerical results (Sec. VIII), the conditions for the validity of GOM do not generally hold in a realistic SL model.

\section{MULTILAYERED SPHERE}

As discussed in Sect. [II] Hilgenfeldt et al. [20, 21] have assumed UBM as well as GOM in deriving the spectral radiance. This simplification serves as an illustration of the essential ingredients in SBSL. Yet its validity has to be verified, and for realistic calculations comparable to the experiment, it is necessary to take full account of the hydrodynamics inside the bubble. In light of this, in the following we will employ CFM developed by Ho et al. 35. to simulate the hydrodynamics of the bubble. It is then obvious that UBM breaks down in such situation and, instead, we can model the inhomogeneous SL bubble as a multilayered sphere (MLS) with a piecewise-constant configuration of temperature and refractive index (see Fig. 2). In realistic calculations, the number of layers is so large that MLS is able to mimic the continuous distribution obtained from CFM. On the other hand, MLS also includes UBM as a special case where there is only a single layer.

Consider an MLS (i.e. the bubble) having a piecewiseconstant temperature profile $T=T_{j}, j=1,2, \ldots, N-1$. As the absorption cross-section simply becomes the sum of the contribution from each layer, it follows directly from Eq. (2.9) that:

$$
P_{\lambda}=\sum_{j=1}^{N-1} B_{\lambda}\left(T_{j}\right) \sigma_{\mathrm{abs}, j}
$$

where $\sigma_{\mathrm{abs}, j}$ is the contribution of the $j^{\text {th }}$ layer to the total absorption cross-section of the MLS. Eqs. (3.3) and (4.1) are the equations that we use for computing the power emitted from the SL bubble. In Sect. [V] we will evaluate $\sigma_{\mathrm{abs}, j}$ from a wave optics perspective.

\section{WAVE OPTICS MODEL}

To evaluate $\sigma_{\mathrm{abs}, j}$ in an MLS, we first determine the electromagnetic field inside the absorbing sphere using the transfer matrix formalism applicable to multilayered configuration (see, e.g. [36]).

Consider a circularly polarized plane wave illuminating an absorbing MLS (see Fig. 2). The MLS is composed of $N-1$ spherical shells, and the refractive index, the inner and outer radii of the $j$-th shell $(j=1,2, \ldots, N-1)$ are $n_{j}, a_{j-1}$ and $a_{j}$, respectively $\left(a_{0}=0\right.$ and $a_{N-1}=R$ are assumed). The extended medium surrounding the MLS (i.e. the bubble) is considered as the $N$-th layer and has a refractive index $n_{N}$.

The electric and magnetic fields of the incident wave are given by $\boldsymbol{E}_{\text {inc }}(\boldsymbol{r})=(\hat{\boldsymbol{x}}+i \hat{\boldsymbol{y}}) \exp (i k z)$ and $\boldsymbol{B}_{\text {inc }}(\boldsymbol{r})=$ $-i \boldsymbol{E}(\boldsymbol{r}) / c$. Hereafter we assume that the wave has positive helicity. The electric and magnetic field inside the $j$-th layer of the MLS $(j=1,2, \ldots, N)$, can be respectively expressed in multipole expansion as:

$$
\begin{aligned}
\boldsymbol{E}_{j}(\boldsymbol{r})= & \sum_{l=1}^{\infty} i^{l} \sqrt{4 \pi(2 l+1)} \\
& \times\left\{f_{j}^{(\mathrm{E})} \boldsymbol{Y}_{l, 1}^{(0)}+\frac{\nabla \times\left[f_{j}^{(\mathrm{M})} \boldsymbol{Y}_{l, 1}^{(0)}\right]}{n_{j} k}\right\}, \\
\boldsymbol{B}_{j}(\boldsymbol{r})= & -\frac{i n_{j}}{c} \sum_{l=1}^{\infty} i^{l} \sqrt{4 \pi(2 l+1)} \\
& \times\left\{f_{j}^{(\mathrm{M})} \boldsymbol{Y}_{l, 1}^{(0)}+\frac{\nabla \times\left[f_{j}^{(\mathrm{E})} \boldsymbol{Y}_{l, 1}^{(0)}\right]}{n_{j} k}\right\},
\end{aligned}
$$

where the vector spherical harmonics $\boldsymbol{Y}_{l m}^{(0)}(\theta, \phi) \equiv$ $-i \boldsymbol{r} \times \nabla Y_{l m}(\theta, \phi) / \sqrt{l(l+1)}$, with $Y_{l m}(\theta, \phi)$ being the ordinary spherical harmonics [40].

For the extended medium where $j=N, f_{N}^{(\mathrm{E})}=f_{N}^{(\mathrm{M})}=$ $j_{l}\left(n_{N} x\right)$, with $j_{l}$ being the spherical Bessel function of the $l$-th order and $x=k r$, Eqs. (5.1)-(5.2) reduce to the ordinary multipole expansion of a plane wave. For $j<N, f_{j}^{(\mu)}(r)(\mu=\mathrm{E}, \mathrm{M})$ are governed by the radial wave equation:

$$
\left[\frac{\mathrm{d}^{2}}{\mathrm{~d} r^{2}}+n_{j}^{2} k^{2}-\frac{l(l+1)}{r^{2}}\right] r f_{j}^{(\mu)}(r)=0,
$$

and, in addition, they satisfy the standard boundary con- 
ditions on electromagnetic fields imposed at $r=a_{j}$ :

$$
\begin{aligned}
& f_{j}^{(\mathrm{E})}(r)=f_{j+1}^{(\mathrm{E})}(r), \\
& \frac{\mathrm{d}}{\mathrm{d} r}\left[r f_{j}^{(\mathrm{E})}\right]=\frac{\mathrm{d}}{\mathrm{d} r}\left[r f_{j+1}^{(\mathrm{E})}(r)\right], \\
& n_{j} f_{j}^{(\mathrm{M})}(r)=n_{j+1} f_{j+1}^{(\mathrm{M})}(r), \\
& \frac{n_{j+1}}{n_{j}} \frac{\mathrm{d}}{\mathrm{d} r}\left[r f_{j}^{(\mathrm{M})}\right]=\frac{\mathrm{d}}{\mathrm{d} r}\left[r f_{j+1}^{(\mathrm{M})}(r)\right] .
\end{aligned}
$$

In general $f_{j}^{(\mu)}(r)$ can be written as:

$$
f_{j}^{(\mu)}(r)=\alpha_{j}^{(\mu)} j_{l}\left(n_{j} k r\right)+\beta_{j}^{(\mu)} h_{l}^{(1)}\left(n_{j} k r\right),
$$

where $h_{l}^{(1)}$ is the $l$-th order spherical Hankel function of the first kind. Substituting Eq. (5.8) into Eqs. (5.4)-(5.7) and recasting them into matrix form, we can show that:

$$
\left[\begin{array}{c}
\alpha_{j+1}^{(\mu)} \\
\beta_{j+1}^{(\mu)}
\end{array}\right]=\mathbf{T}_{j}^{(\mu)}\left[\begin{array}{c}
\alpha_{j}^{(\mu)} \\
\beta_{j}^{(\mu)}
\end{array}\right]
$$

where $\mathbf{T}_{j}^{(\mathrm{E})}$ and $\mathbf{T}_{j}^{(\mathrm{M})}$ are respectively the TE-mode and TM-mode transfer matrix at $r=a_{j}$, given explicitly by:

$$
\begin{aligned}
& \mathbf{T}_{j}^{(\mathrm{E})}=-i n_{j+1} x_{j}^{2} \times \\
& {\left[\begin{array}{cc}
W_{j+1, j}^{(\mathrm{E})}\left[j_{l}\left(n_{j} x_{j}\right), h_{l}^{(1)}\left(n_{j+1} x_{j}\right)\right] & W_{j+1, j}^{(\mathrm{E})}\left[h_{l}^{(1)}\left(n_{j} x_{j}\right), h_{l}^{(1)}\left(n_{j+1} x_{j}\right)\right] \\
-W_{j+1, j}^{(\mathrm{E})}\left[j_{l}\left(n_{j} x_{j}\right), j_{l}\left(n_{j+1} x_{j}\right)\right] & -W_{j+1, j}^{(\mathrm{E})}\left[h_{l}^{(1)}\left(n_{j} x_{j}\right), j_{l}\left(n_{j+1} x_{j}\right)\right]
\end{array}\right],} \\
& \mathbf{T}_{j}^{(\mathrm{M})}=-i n_{j+1}^{2} n_{j} x_{j}^{2} \times \\
& {\left[\begin{array}{cl}
W_{j+1, j}^{(\mathrm{M})}\left[j_{l}\left(n_{j} x_{j}\right), h_{l}^{(1)}\left(n_{j+1} x_{j}\right)\right] & W_{j+1, j}^{(\mathrm{M})}\left[h_{l}^{(1)}\left(n_{j} x_{j}\right), h_{l}^{(1)}\left(n_{j+1} x_{j}\right)\right] \\
-W_{j+1, j}^{(\mathrm{M})}\left[j_{l}\left(n_{j} x_{j}\right), j_{l}\left(n_{j+1} x_{j}\right)\right] & -W_{j+1, j}^{(\mathrm{M})}\left[h_{l}^{(1)}\left(n_{j} x_{j}\right), j_{l}\left(n_{j+1} x_{j}\right)\right]
\end{array}\right] .}
\end{aligned}
$$

Here $x_{j}=k a_{j}$, and for convenience we define the generalized Wronskian $W_{j+1, j}^{(\mu)}[f, g]$ for TE and TM modes as:

$$
\begin{aligned}
W_{j+1, j}^{(\mathrm{E})}[f, g]= & f g^{\prime}-f^{\prime} g, \\
W_{j+1, j}^{(\mathrm{M})}[f, g]= & \frac{f g^{\prime}}{n_{j+1}^{2}}-\frac{f^{\prime} g}{n_{j}^{2}} \\
& +\left(\frac{1}{n_{j+1}^{2}}-\frac{1}{n_{j}^{2}}\right) \frac{f g}{x},
\end{aligned}
$$

where $^{\prime}=\mathrm{d} / \mathrm{d} x$.

In short, the transfer matrix $\mathbf{T}_{j}^{(\mu)}$ can be written as:

$$
\mathbf{T}_{j}^{(\mu)}=\left[\begin{array}{ll}
A_{j}^{(\mu)} & B_{j}^{(\mu)} \\
C_{j}^{(\mu)} & D_{j}^{(\mu)}
\end{array}\right]
$$

We can now solve for the field coefficients $\alpha_{j}^{(\mu)}$ and $\beta_{j}^{(\mu)}$ using the regularity condition at $r=0$ and radiation boundary conditions at $r=\infty$, leading to the following relation:

$$
\left[\begin{array}{c}
1 \\
\beta_{N}^{(\mu)}
\end{array}\right]=\left[\begin{array}{ll}
A^{(\mu)} & B^{(\mu)} \\
C^{(\mu)} & D^{(\mu)}
\end{array}\right]\left[\begin{array}{c}
\alpha_{1}^{(\mu)} \\
0
\end{array}\right],
$$

Here $A^{(\mu)}, B^{(\mu)}, C^{(\mu)}, D^{(\mu)}$ (without the subscript $j$ ) are the elements of the total transfer matrix $\mathbf{T}^{(\mu)}$ from the layer $j=1$ to the layer $j=N-1$, i.e. $\mathbf{T}^{(\mu)}=$ $\mathbf{T}_{N-1}^{(\mu)} \mathbf{T}_{N-2}^{(\mu)} \cdots \mathbf{T}_{1}^{(\mu)}$. From Eq. (5.15) we immediately get

$$
\begin{aligned}
\alpha_{1}^{(\mu)} & =\left(A^{(\mu)}\right)^{-1}, \\
\beta_{N}^{(\mu)} & =C^{(\mu)} \alpha_{1}^{(\mu)}=C^{(\mu)} / A^{(\mu)} .
\end{aligned}
$$

The field coefficients of each layer $\alpha_{j}^{(\mu)}, \beta_{j}^{(\mu)}$ is now readily obtained by iteratively applying Eq. (5.9), from $j=1$ to $j=N-1$, using the boundary conditions Eqs. (5.16)(5.17).

Now we proceed to calculate the absorption crosssection of the $j$-th spherical shell. To this end, we first evaluate:

$$
\mathcal{F}_{j}=\frac{n_{N} c a_{j}^{2}}{2} \operatorname{Re}\left\{\int \mathrm{d} \Omega \boldsymbol{E}_{j} \cdot\left(\hat{\boldsymbol{r}} \times \boldsymbol{B}_{j}^{*}\right)\right\},
$$

which is directly proportional to the energy flux $\mathcal{F}_{j}$ crossing the $j$-th interface at $r=a_{j}$. With Eqs. (5.1), (5.2), we find, after some algebraic manipulations,

$$
\begin{aligned}
\mathcal{F}_{j}= & \frac{2 \pi x n_{N}}{k^{2}} \operatorname{Im}\left\{n_{j}^{*} \sum_{l=1}^{\infty}(2 l+1) \times\right. \\
& \left.\left\{\frac{f_{j}^{(E)}\left[x f_{j}^{(E)}\right]^{\prime *}}{n_{j}^{*}}-\frac{f_{j}^{(M) *}\left[x f_{j}^{(M)}\right]^{\prime}}{n_{j}}\right\}\right\},
\end{aligned}
$$


where $x$ is evaluated at $r=a_{j}$. Following directly from energy conservation, the absorption cross-section of the $j$-th spherical shell, $\sigma_{\mathrm{abs}, j}$ is given by the difference between $\mathcal{F}_{j}$ and $\mathcal{F}_{j-1}$ :

$$
\sigma_{\mathrm{abs}, j}=\mathcal{F}_{j}-\mathcal{F}_{j-1} .
$$

Eqs. (5.19)-(5.20) in conjunction with Eqs. (2.12) and (4.1), with no simplifying assumptions based on geometric optics and optical thickness, are the major results of the wave optics model (WOM) introduced here, which will be used in this paper to calculate the light emission of a SL bubble.

\section{PLASMA MODEL OF MULTILAYERED SPHERE}

\section{A. Formation of Plasma}

In order to consider light emission processes in an MLS, we have to find the complex refractive index $n_{j}$ and hence the absorption coefficient $\kappa_{j}$ of each layer. In the presence of the high temperature developed during the collapse of a SL bubble, the gas inside the bubble is partially ionized at the instant of light emission 13, 19, 20, 21, 35, 36]. Here we adopt a simple collision-dominated plasma model in which the collision frequency $\nu$ is a constant dependent on the concentration and temperature of the plasma [37]. The refractive index is then given by:

$$
n^{2}(\omega)=n_{b}^{2}-\frac{\omega_{p}^{2}}{\omega^{2}+i \nu \omega}
$$

where $\omega_{p}=\sqrt{N_{e} e^{2} / m \epsilon_{0}}$ is the plasma frequency,

$$
n_{b}=\left(\frac{1+2 N_{0} \alpha / 3 \epsilon_{0}}{1-N_{0} \alpha / 3 \epsilon_{0}}\right)^{\frac{1}{2}},
$$

is the contribution to the refractive index due to the background neutral atoms as given by the Clausius-Mossotti equation (see, e.g. 41] ), and $\alpha$ is the atomic polarizability, with $e$ and $m$ being the charge and the mass of electron, respectively. For convenience we have suppressed the $j$-dependence of the relevant physical quantities in the above equations. In the subsequent discussion we will use CFM developed in [35] to determine the number densities of electron and atom, $N_{e}$ and $N_{0}$, as a function of time.

\section{B. Collision Processes in plasma}

Around the instant of maximum compression, the temperature of a sonoluminescing bubble could reach tens of thousands of kelvins, ionizing the gas content inside. While Ref. 20] showed that the fraction of $\mathrm{Ar}^{+}$ions amounts to less than $1 \%$ of the bubble content using a uniform bubble assumption; Ref. [35] took account of full hydrodynamics and showed that this fraction can be as large as $30 \%$. However, both papers point to the fact that the bubble content becomes a partially ionized plasma with Ar being the dominant species. Under this condition, bremsstrahlung is thought to play a major role in the light emission mechanism; in particular, electronatom bremsstrahlung is expected to be the dominant process compared to other bremsstrahlung processes [20, 21].

On the other hand, as the numerical results obtained from CFM showed the degree of ionization may be much higher than those from UBM, we have developed here the WOM to take account of effects due to reflection, refraction and diffraction as well. In accordance with the approach of WOM, one has to consider the total effective collision frequency $\nu$, which is the sum of contributions from electron-ion collision, electron-atom collision and electron-ion recombination. According to the initial and final states of the electron, the first two are also known as free-free transitions, the latter one as free-bound transition. Of course, the direct product of such collisions is the emission of photons and the corresponding mechanisms are electron-ion bremsstrahlung, electron-atom bremsstrahlung as well as electron-ion recombination. Therefore, one can easily see the difference, as well as the relation between our approach and that proposed in 20, 21. In the following we provide the formulas of the collision frequencies in these processes.

\section{Electron-ion collision}

The simplest picture describing electron-ion collisions and electron-ion bremsstrahlung is to regard them as individual binary events so that collective phenomena do not enter. Under such an assumption the differential emission cross-section $d \sigma_{f}$, which measures the probability of light emission due to the scattering of a unit incident electron flux from an ion with charge $Z e$, is given by the well-known Kramer's formula [39]. It is customary to include quantum mechanical corrections to this classical formula as the Gaunt factor [37]. For free-free transitions, including the free-free Gaunt factor $g_{\mathrm{ff}}(f, v)$ gives $d \sigma_{f}$ as:

$$
\frac{\mathrm{d} \sigma_{f}}{\mathrm{~d} f}=\left(\frac{e^{2}}{4 \pi \epsilon_{0} \hbar c}\right)^{3} \frac{16 \pi \hbar^{2}}{3 \sqrt{3} m^{2} v^{2}} \frac{Z^{2}}{f} g_{\mathrm{ff}}(f, v),
$$

where $f$ is the light frequency and $v$ is the speed of the incident electron. Within the range of optical frequencies, the free-free Gaunt factor is usually of the order 1.

The collisions between charged particles is formally described by the scattering cross-section $\sigma(\theta)$, measuring the probability of scattering at an angle $\theta$ of a unit incident electron flux from an ion. However, the crosssection used in transport theory to predict the scattering frequency is $\sigma_{\mathrm{tr}}$, related to the former through the relation $\sigma_{\mathrm{tr}}=\overline{\sigma(\theta)}(1-\overline{\cos \theta})$, where the over-bar indicates 
averaging over the scattering angle $\theta$. Using the relation between the differential emission cross-section $\mathrm{d} \sigma_{f}$ and the transport cross-section $\sigma_{\operatorname{tr}}$ 39]

$$
\frac{\mathrm{d} \sigma_{f}}{\mathrm{~d} f}=\frac{8}{3} \frac{e^{2} v^{2}}{4 \pi \epsilon_{0} c^{3} h f} \sigma_{\mathrm{tr}},
$$

the transport cross-section is found to be

$$
\sigma_{\mathrm{tr}}=\frac{4 \pi^{2}}{\sqrt{3}}\left(\frac{Z e^{2}}{4 \pi \epsilon_{0} m v^{2}}\right)^{2} g_{\mathrm{ff}}(f, v) .
$$

The collision frequency, defined as $\nu=N v \sigma_{\mathrm{tr}}$, where $N$ is the number density of the background species $\left(N_{i}\right.$ for ions or $N_{0}$ for atoms), is then

$$
\nu_{\mathrm{ei}}=\frac{4 \pi^{2}}{\sqrt{3}} \frac{N_{i} Z^{2} e^{4}}{\left(4 \pi \epsilon_{0}\right)^{2} m^{2} v^{3}} g_{\mathrm{ff}}(f, v) .
$$

\section{Electron-ion Recombination}

In electron-ion recombination, an electron is first captured by an ion, forming a bound state with energy levels labelled by quantum number $n$. A photon is released in such a process and the differential emission cross-section is given by Eq. (6.3) with the free-free Gaunt factor replaced by the free-bound Gaunt factor, $g_{\mathrm{fb}}(n, f, v)$. In addition to its dependence on the photon frequency $f$ and the velocity $v$ of the incident electron, the freebound Gaunt factor is also a function of $n$ and approximately of the order unity in optical frequencies. Accordingly, the transport cross-section and the electron-ion recombination collision frequency are given respectively by Eqs. 6.5 and (6.6) with $g_{\mathrm{fb}}(n, f, v)$ replacing $g_{\mathrm{ff}}(f, v)$.

\section{Electron-atom collision}

An electron moving near a neutral atom can also experience a short-range Coulomb field, emitting radiation commonly known as electron-atom bremsstrahlung. There is no simple theory to determine the corresponding transport cross-section as we are aware of; and common practice is to determine it from experiment with different incident electron energies. With good accuracy in the relevant range of electron energies for an argon SL bubble, $\sigma_{\mathrm{tr}}$ has a linear dependence on the electron energy $E_{e}=m v^{2} / 2$ and $\sigma_{\mathrm{tr}}=c_{\mathrm{tr}} m v^{2} / 2+d_{\mathrm{tr}}$, with the empirical constants $c_{\mathrm{tr}} \simeq 0.1 \mathrm{~m}^{2} \mathrm{~J}^{-1}$ and $d_{\mathrm{tr}} \simeq-0.6 \times 10^{-20} \mathrm{~m}^{2}$ $[20,42]$. The electron-atom collision frequency is therefore

$$
\nu_{\mathrm{ea}}=N_{0} v\left(c_{\mathrm{tr}} m v^{2} / 2+d_{\mathrm{tr}}\right)
$$

\section{Effective collision frequency}

Assuming local thermodynamic equilibrium prevails in the plasma (as in the case for SL) and weak damping $(\nu \ll \omega)$, we proceed to calculate the effective collision frequency [37] defined by $\nu^{\text {eff }}=\bar{\nu}=N \overline{v \sigma_{\operatorname{tr}}(v)}$, here $\cdots$ indicates the Maxwellian average:

$$
\begin{aligned}
\cdots= & \frac{4 \pi}{3}\left(\frac{m}{2 \pi k_{B} T}\right)^{\frac{3}{2}}\left(\frac{m}{k_{B} T}\right) \\
& \times \int_{0}^{\infty} \mathrm{d} v v^{4} e^{-m v^{2} / 2 k_{B} T}(\cdots) .
\end{aligned}
$$

Accordingly, the effective collision frequencies for electron-ion bremsstrahlung, electron-ion recombination and electron-atom bremsstrahlung are obtained as (for clarity we drop the superscript 'eff'):

$$
\begin{aligned}
\nu_{\mathrm{ei}(\mathrm{rc})}= & 2\left(\frac{2 \pi}{3}\right)^{\frac{3}{2}} N_{i}\left(\frac{Z e^{2}}{4 \pi \epsilon_{0} k_{B} T}\right)^{2}\left(\frac{k_{B} T}{m}\right)^{\frac{1}{2}} \\
& \times e^{-h f / k_{B} T} \frac{\overline{g_{\mathrm{ff}(\mathrm{fb})}}(f, T)}{3} N_{0}\left(\frac{k T}{\pi m}\right)^{\frac{1}{2}}\left(3 c_{\mathrm{tr}} k_{B} T+d_{\mathrm{tr}}\right)
\end{aligned}
$$

Here the exponential factor $\exp \left(-h f / k_{B} T\right)$ is commonly referred to as the Cillie exponential factor 43$] ; \overline{g_{\mathrm{ff}}}(f, T)$ and $\overline{g_{\mathrm{fb}}}(f, T)$ are the velocity-averaged free-free and freebound Gaunt factor respectively 44]:

$$
\begin{aligned}
& \overline{g_{\mathrm{ff}}}(f, T)=\frac{e^{h f / k_{B} T}}{k_{B} T} \int_{h f}^{\infty} \mathrm{d} E g_{\mathrm{ff}}(f, E) e^{-E / k_{B} T}(, 6 \\
& \overline{g_{\mathrm{fb}}}(f, T)=2 x_{1} \sum_{n^{*}}^{\infty} \frac{1}{n^{3}} e^{x_{n}} g_{\mathrm{fb}}(n, f, v) .
\end{aligned}
$$

In (6.12), $x_{n}=E_{\text {ion }} / n^{2} k_{B} T$, with $E_{\text {ion }}$ being the first ionization energy of the atom, and $n^{*}$ is the lowest level for which $E_{n^{*}}<h f$.

Note that, assuming $g_{\mathrm{ff}}, g_{\mathrm{fb}} \simeq 1$, the above equations can be reduced to

$$
\begin{aligned}
\overline{g_{\mathrm{ff}}}(f, T) & =1, \\
\overline{g_{\mathrm{fb}}}(f, T) & =2 x_{1} \sum_{n^{*}}^{\infty} \frac{1}{n^{3}} e^{x_{n}} .
\end{aligned}
$$

The summation in Eq. (6.14) can be further simplified if the photon energy is small compared with the ionization energy, so that the energy level $n^{*}$ is high in comparison with the ground state, as is the case for argon 20]. Since the density of the levels increases rapidly with increasing $n$, the discrete levels higher than $n^{*}$ can be replaced by a continuous spectrum and the summation replaced by an integration, and Eq. (6.14) simplifies to

$$
\begin{aligned}
\overline{g_{\mathrm{fb}}}(f, T) & =e^{h f / k_{B} T}-1 \\
& =e^{h c / k_{B} T \max \left\{\lambda, \lambda_{2}\right\}}-1 .
\end{aligned}
$$

As the absorption coefficient $\kappa$ is related to the effective collision frequency as:

$$
\kappa=\left(\frac{\omega_{p}}{\omega}\right)^{2} \frac{\nu}{c}
$$


we can find the absorption coefficients corresponding to Eqs. (6.9)-(6.10):

$$
\begin{aligned}
\kappa_{\mathrm{ei}(\mathrm{rc})}= & \frac{4}{3}\left(\frac{2 \pi}{3 m k_{B} T}\right)^{\frac{1}{2}} \frac{Z^{2} N_{i}^{2} e^{6} \lambda^{2}}{\left(4 \pi \epsilon_{0}\right)^{3} k_{B} T c^{3} m} \\
& \left.\times e^{-h f / k_{B} T} \frac{N_{\mathrm{ff}(\mathrm{fb})}}{f}, T\right), \\
\kappa_{\mathrm{ea}}= & 8 \sqrt{2} \frac{N_{i} N_{0} e^{2} \lambda^{2}}{4 \pi \epsilon_{0} c^{3}}\left(\frac{k_{B} T}{\pi m}\right)^{\frac{3}{2}} \\
& \times\left(c_{t r}+\frac{d_{t r}}{3 k_{B} T}\right),
\end{aligned}
$$

The absorption coefficient $\kappa_{\mathrm{ei}(\mathrm{rc})}$ in Ref. 20] differs from ours in two ways. (i) They differ by a factor of $k_{B} T / h f$. However, this difference is not very significant as $k_{B} T \simeq$ $h f$ in SL; (ii) For electron-ion bremsstrahlung, the freefree Gaunt factor and the Cillie exponential correction were neglected in Ref. [20]. As we will discuss later (see Fig. (7), this could result in a factor of 3 difference in the calculated spectra.

In the present paper, we explicitly take account of all Gaunt factors and the exponential correction, in particular we adopt the fitting formula proposed by Itoh et al. 45, 46] to compute the average free-free Gaunt factor. For the free-bound Gaunt factor, since the photon energies $1.5-6.2 \mathrm{eV}$, corresponding to the wavelength $200-800 \mathrm{~nm}$, is small compared with the ionization energy $\left(E_{\text {ion }}=15.8 \mathrm{eV}\right.$ for argon), Eq. 6.15) is still a good approximation and we retain it for computing the average free-bound Gaunt factor. As a remark, following Eqs. (6.9), (6.13) and 6.15) we have $\nu_{\mathrm{rc}} / \nu_{\mathrm{ei}} \simeq e^{h f / k_{B} T}-1$, hence electron-ion bremsstrahlung is more dominant over recombination when $h f \ll k_{B} T$. For SL, however, both processes are important since the thermal energy is typically $\sim 1.7-4.3 \mathrm{eV}$.

For the purpose of comparison, in the following discussion we will use two different sets of formulas, respectively denoted by P1 and P2 models, to calculate the collision frequencies. The P1 model employs the free-bound Gaunt factor, but ignore the free-free Gaunt factor and the Cillie exponential cut-off factor:

$$
\begin{aligned}
\nu_{\mathrm{ei}}= & 2\left(\frac{2 \pi}{3}\right)^{\frac{3}{2}} N_{i}\left(\frac{Z e^{2}}{4 \pi \epsilon_{0} k_{B} T}\right)^{2}\left(\frac{k_{B} T}{m}\right)^{\frac{1}{2}} \frac{k_{B} T}{h f} \\
\nu_{\mathrm{rc}}= & 2\left(\frac{2 \pi}{3}\right)^{\frac{3}{2}} N_{i}\left(\frac{Z e^{2}}{4 \pi \epsilon_{0} k_{B} T}\right)^{2}\left(\frac{k_{B} T}{m}\right)^{\frac{1}{2}} \frac{k_{B} T}{h f} \\
& \times \overline{g_{\mathrm{fb}}}(f, T) \\
\nu_{\mathrm{ea}}= & \frac{8 \sqrt{2}}{3} N_{a}\left(\frac{k_{B} T}{\pi m}\right)^{\frac{1}{2}}\left(3 c_{t r} k_{B} T+d_{t r}\right) .
\end{aligned}
$$

Through (6.16), it is obvious that this set of formulas for the collision frequencies are consistent with the set of absorption coefficients used in [20, 21].

By contrast, the P2 model, derived earlier in this section, readily takes account of free-bound Gaunt factor, free-free Gaunt factor and exponential cut-off factor:

$$
\begin{aligned}
\nu_{\mathrm{ei}}= & 2\left(\frac{2 \pi}{3}\right)^{\frac{3}{2}} N_{i}\left(\frac{Z e^{2}}{4 \pi \epsilon_{0} k_{B} T}\right)^{2}\left(\frac{k_{B} T}{m}\right)^{\frac{1}{2}} \\
& \times e^{-h f / k_{B} T} \overline{\overline{g_{\mathrm{ff}}}}(f, T), \\
\nu_{\mathrm{rc}}= & 2\left(\frac{2 \pi}{3}\right)^{\frac{3}{2}} N_{i}\left(\frac{Z e^{2}}{4 \pi \epsilon_{0} k_{B} T}\right)^{2}\left(\frac{k_{B} T}{m}\right)^{\frac{1}{2}} \\
& \times e^{-h f / k_{B} T} \overline{g_{\mathrm{fb}}}(f, T), \\
\nu_{\mathrm{ea}}= & \frac{8 \sqrt{2}}{3} N_{a}\left(\frac{k_{B} T}{\pi m}\right)^{\frac{1}{2}}\left(3 c_{t r} k_{B} T+d_{t r}\right) .
\end{aligned}
$$

Finally we state the basic assumptions underpinning the above formulas for plasma collision processes: (1) the plasma is 'cold' meaning that the electron thermal velocity is negligible with respect to the phase velocity of the wave, $v_{\mathrm{th}} \ll v_{\mathrm{ph}}$; (2) the plasma is in the weak coupling regime, i.e. the ions are weakly interacting during their thermal motions. It is customary to indicate the degree of coupling by the dimensionless ion-coupling parameter (see, e.g. [4]]):

$$
\Gamma=\frac{Z^{2} e^{2}}{4 \pi \epsilon_{0} R_{\text {ion }} k_{B} T},
$$

with $R_{\text {ion }}=\left(4 \pi N_{i} / 3\right)^{-\frac{1}{3}}$ being the mean inter-ionic distance. If $\Gamma \ll 1$, the system is said to be weakly coupled; on the other hand if $\Gamma \gtrsim 1$, the system is in the strong coupling regime 47. We find $\Gamma \lesssim 1$ in a typical SL bubble (Sec. VIII), hence the assumption of weak coupling is at least approximately satisfied.

\section{COMPUTATIONAL FLUID MECHANICS}

In this section, we summarize the CFM used in the present paper, which was developed by Cheng et al. 14, 15] and later extended by Ho et al. 35] to include the ionization and recombination processes. The model couples the Rayleigh-Plesset (RP) equation for the bubble wall with the Navier-Stokes (NS) equations for the gas (including all the charged species resulting from ionizations), while independently solving the energy equation for the surrounding water. The number densities of the charged species are computed from the reaction rates approach. The effects of viscosity, surface tension, equation of state (EOS), compressibility and thermal conductivity of the ambient liquid are also taken into account.

\section{A. Bubble-wall dynamics}

To account for the effect of liquid compressibility, a more robust $\mathrm{RP}$ equation that gives the bubble radius $R$ as a function of time $t$ is used [14, 15, 35, 48]:

$$
\frac{1-M}{1+M} R \ddot{R}+\frac{3-M}{2(1+M)} \dot{R}^{2}=H_{l}-\frac{P_{s}\left(t^{\prime}\right)}{\rho_{0}}+\frac{t_{R} \dot{H}_{l}}{1+M} .
$$


Here $t_{R} \equiv R / c_{l}$, with $c_{l}$ the speed of sound in the surrounding liquid, $M \equiv \dot{R} / c_{l}, t^{\prime} \equiv t+t_{R}, \rho_{0}$ is the ambient liquid density, and $P_{s}\left(t^{\prime}\right)=-P_{a} \sin \left(\omega t^{\prime}\right)$ is the sonic driving pressure with frequency $\omega$ and amplitude $P_{a}$. Also, the enthalpy $H_{l}$ and the speed of sound of the liquid and $c_{l}$ are given by:

$$
\begin{aligned}
H_{l} & =\int_{P_{0}}^{P_{l}} \frac{\mathrm{d} P_{l}}{\rho_{l}}, \\
c_{l}^{2} & =\frac{\mathrm{d} P_{l}}{\mathrm{~d} \rho_{l}} .
\end{aligned}
$$

This modified RP equation includes terms to first order in the Mach number $M$ of the bubble wall and allows for a variable $c_{l}$.

Combining Eqs. (7.2), (7.3) with the EOS of the ambient liquid in the modified Tait form 49],

$$
\frac{P_{l}+B}{P_{0}+B}=\left(\frac{\rho_{l}}{\rho_{0}}\right)^{n}
$$

yields the explicit forms for $H_{l}$ and $c_{l}$ :

$$
\begin{aligned}
H_{l} & =\frac{n}{n-1}\left(\frac{P_{l}+B}{\rho_{l}}-\frac{P_{0}+B}{\rho_{0}}\right), \\
c_{l} & =\frac{n\left(P_{l}+B\right)}{\rho_{l}},
\end{aligned}
$$

where $B=3049.13$ bar and $n=7.15$ are valid for water up to $10^{5}$ bar.

Eqs. (7.1), (7.5) and (7.6) must be supplemented by the boundary condition at the bubble wall, namely, that the pressure $P_{l}(t)$ on the liquid side of the gas-liquid interface differs from the pressure $P(R, t)$ on the gas side of the gas-liquid interface by the effects of surface tension and the normal component of viscous stresses [49],

$$
P(R, t)-\left.\tau_{r r}\right|_{r=R}=P_{l}(t)+\frac{4 \eta_{l} \dot{R}}{R}+\frac{2 \sigma}{R} .
$$

\section{B. Hydrodynamics of gas}

The conservation of mass, momentum and energy for the gas flow in the spherical bubble is described by the compressible NS equations. They can be rewritten into a conservative form with source terms as:

$$
\begin{gathered}
\frac{\partial \rho}{\partial t}+\frac{\partial}{\partial r}(\rho v)=-\frac{2 \rho v}{r}, \\
\frac{\partial(\rho v)}{\partial t}+\frac{\partial}{\partial r}\left(\rho v^{2}+P\right) \\
=-\frac{2 \rho v^{2}}{r}+\frac{1}{r^{2}} \frac{\partial}{\partial r}\left(r^{2} \tau_{r r}\right)+\frac{\tau_{r r}}{r},
\end{gathered}
$$

$$
\begin{aligned}
& \frac{\partial(\rho E)}{\partial t}+\frac{\partial}{\partial r}(\rho E+P) v \\
= & -\frac{2(\rho E+P) v}{r}+\frac{1}{r^{2}} \frac{\partial}{\partial r}\left[r^{2}\left(v \tau_{r r}+k \frac{\partial T}{\partial r}\right)\right] .
\end{aligned}
$$

Here $r, \rho, v, P, T, \tau_{r r}, k e$ and $E=e+v^{2} / 2$ are the radial distance from the center of the bubble, gas density, radial velocity, pressure, temperature, normal viscous stress, coefficient of thermal conductivity, the internal energy and total energy per unit mass, respectively.

If, due to ionizations and recombinations, there exists $N_{s}$ species inside the bubble, then $N_{s}-1$ mass conservation equations for these species must be supplemented with Eqs. (7.8)-(7.10). In Ref. 35], the maximum ionization level of the gas atom is taken to be 5 , making a total of $N_{s}=7$ species inside the bubble. This is more than adequate for the present temperature range; in fact, Ho et al. 35] have shown that even the second ionization level can be safely ignored in practice. Note, since the ion densities change due to ionizations and recombinations, source terms must be added to the right hand side of the conservation equations.

For convenience, let $f_{j}$ be the mass fraction of $\mathrm{Ar}^{j+}$ (with $j=0,1,2,3,4,5$ ) or electrons (with $j=e$ ), so that $\sum_{j=e, 0}^{5} f_{j}=1$. Therefore $\rho f_{j}$ represents the mass density of an individual species. The number density of an individual species is related to its mass fraction by $n_{j}=\rho f_{j} / m_{j}$, where $m_{j}$ is the mass of an atom $(j=0)$, or an ion with a charge $j(j=1-5)$, or an electron $(j=e)$. The mass conservation equations of the species is then given by

$$
\frac{\partial\left(\rho f_{j}\right)}{\partial t}+\frac{\partial}{\partial r}\left(\rho f_{j} v\right)=-\frac{2 \rho f_{j} v}{r}+\left(S_{s}\right)_{j} .
$$

Here, the extra term $\left(S_{s}\right)_{j}$ is the source term for $\rho f_{j}$ which arises from ionization and recombination processes. It depends on the net rate of change of the number density of the species $\dot{n}_{j}$ through:

$$
\left(S_{s}\right)_{j}=m_{j} \dot{n}_{j} .
$$

For the ions $(j=0,1,2,3,4,5)$, the net rate of change is given by:

$$
\begin{aligned}
\dot{n_{j}}= & n_{j-1} n_{e} \alpha_{j-1 \rightarrow j}^{\text {ion }}-n_{j} n_{e} \alpha_{j \rightarrow j+1}^{\text {ion }} \\
& +n_{j+1} n_{e}\left(\alpha_{j+1 \rightarrow j}^{\text {rrec }}+\alpha_{j+1 \rightarrow j}^{\text {trec }}\right) \\
& -n_{j} n_{e}\left(\alpha_{j \rightarrow j-1}^{\text {rrec }}+\alpha_{j \rightarrow j-1}^{\text {trec }}\right),
\end{aligned}
$$

where $\alpha_{j \rightarrow j+1}^{\text {ion }}, \alpha_{j \rightarrow j-1}^{\text {rrec }}$ and $\alpha_{j \rightarrow j-1}^{\text {trec }}$ are the rates of ionization, radiative recombination and three-body recombination of particles with a charge of $j$, respectively. The formulas for these rates can be found in [50].

The net rate of change of the number density of electrons is simply given by charge conservation. Now that

$$
f_{e}=1-\sum_{j=0}^{5} f_{j},
$$


taking time derivative and multiplying both sides by $\rho$ gives:

$$
\left(S_{s}\right)_{e}=-\sum_{j=0}^{5}\left(S_{s}\right)_{j} .
$$

\section{Equation of State of gas}

The hydrodynamics of the bubble is certainly affected by the EOS. The most widely-used van der Waals EOS can be modified to take into account the ionization processes [19]:

$$
\begin{gathered}
P=\left(\sum_{j=0}^{5} \frac{f_{j}}{m_{j}}+\frac{f_{e}}{m_{e}}\right) \frac{k_{B} \rho T}{1-b \rho} \\
e=\frac{3}{2} k_{B} T\left(\sum_{j=0}^{5} \frac{f_{j}}{m_{j}}+\frac{f_{e}}{m_{e}}\right)+k_{B} \sum_{j=1}^{5} \sum_{i=j}^{5} \frac{f_{i}}{m_{i}} T_{j},
\end{gathered}
$$

where $T_{j}$ is the ionization energy of an ion with charge $j-1$, and $b$ the excluded volume. This EOS is denoted as MVEOS.

The physical meanings of the MVEOS, Eqs. (7.16) and (7.17), are manifest. The total pressure $P$ is the sum of the contributions by different species, which are separately taken into account in proportion to their abundances. The internal energy $e$ of the gas consists of both the thermal energy (the first term) and ionization energy (the second term).

\section{Energy transport in the liquid}

The changes in the liquid temperature $T_{l}$ is accounted for with the assumption that the liquid compressibility and viscosity do not affect the heat transfer process between the bubble and the surrounding water. As such, the energy equation for the water is:

$$
\frac{\partial T_{l}}{\partial t}+v_{l} \frac{\partial T_{l}}{\partial r}=D_{l} \frac{1}{r^{2}} \frac{\partial}{\partial r}\left(r^{2} \frac{\partial T_{l}}{\partial r}\right)
$$

where $v_{l}$ and $D_{l}$ are the velocity and thermal diffusion coefficient of the liquid, respectively.

\section{NUMERICAL RESULTS}

In order to elucidate the significance of individual physical factors affecting SBSL, in the following we present and compare numerical results obtained from simulations constructed with different models. Specifically, we consider (i) UBM versus CFM model; (ii) GOM

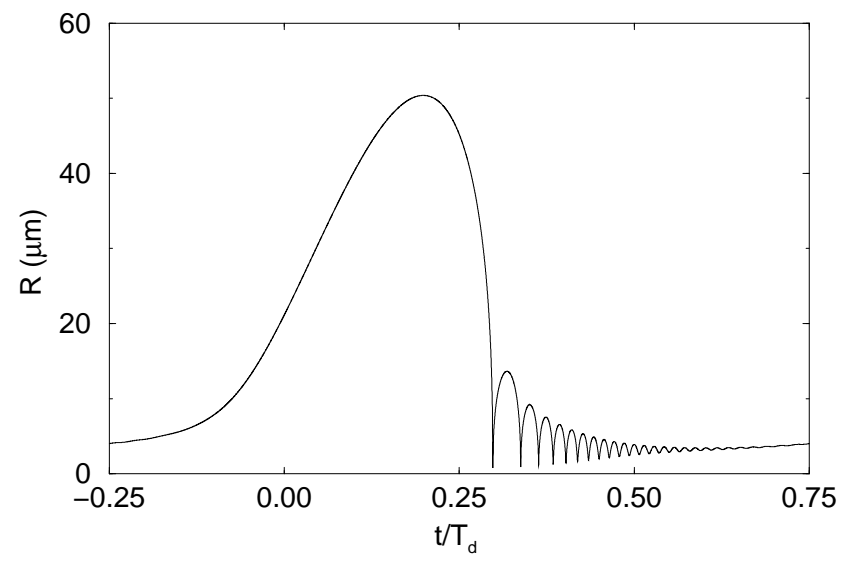

FIG. 3: A plot of the bubble radius $R$ versus time $t$, which is normalized with respect to the acoustic period $T_{\mathrm{d}}$.

versus WOM; and (iii) the two plasma models, P1 versus P2.

The rest of this section is organized as follows: first we study how the effects of various physical entities, including plasma, wave and temperature, can affect the emitted light pulse using the simple UBM model. Then we use the CFM model that is more realistic to mimic SBSL and compare relevant numerical results with those of UBM.

\section{A. Emission in Uniform Bubble Model}

UBM here refers to the model used in [20, 21], where the RP equation assumed incompressibility of the surrounding liquid and a variable polytropic exponent $\gamma(R, \dot{R}, T)$ was used to account for effects of thermal conduction. However, instead of using the fitting formula in [20] for computing the polytropic exponent $\gamma(R, \dot{R}, T)$, we employed the formula proposed in [51] in the simulations. As in Ref. [20], we studied the oscillations of a bubble with ambient radius $R_{0}=5.0 \mu \mathrm{m}$, subjected to an ultrasonic wave with $f=20 \mathrm{kHz}$ and $P_{a}=1.3 \mathrm{~atm}$. Figs. 31 4 respectively show for the UBM the time evolutions of the bubble radius $R$ over one acoustic cycle and, in the vicinity of the maximum bubble compression, the radius and the polytropic exponent $\gamma$. The number density of argon atoms and the temperature near the instant of minimum radius are shown in Fig. 5 . Here we remark that the temperature profile is slightly different from that in [20] due to the difference in the formulas for $\gamma(R, \dot{R}, T)$. The profile remains essentially the same, but the peak temperature in our result is about $3000 \mathrm{~K}$ higher. The temperature and density profiles are used as inputs in our calculations of the spectral radiance, from which other light emission properties (e.g. pulse shapes and FWHM) are obtained. 


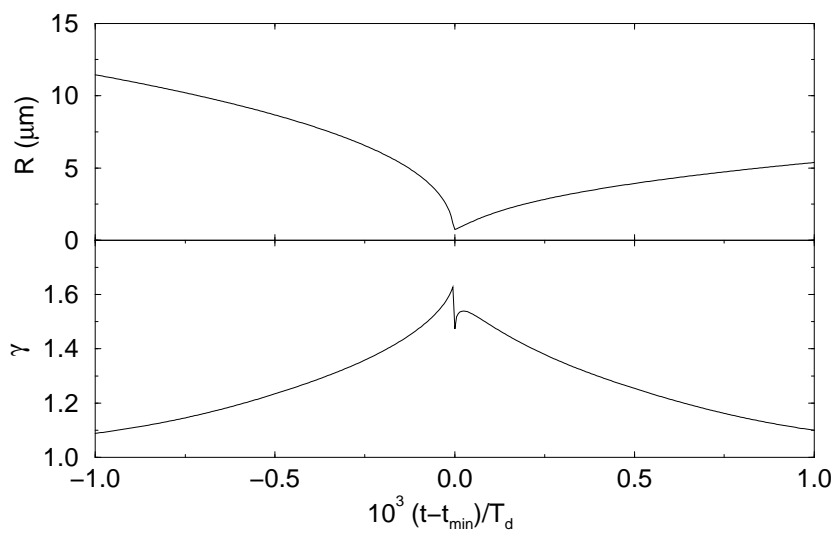

FIG. 4: The bubble radius $R$ (upper panel) and the polytropic exponent $\gamma$ (lower panel) are plotted against the normalized time $10^{3}\left(t-t_{\min }\right) / T_{d}$, where $t_{\min }$ is the moment at which $R$ attains its minimum value of about $0.7 \mu \mathrm{m}$.

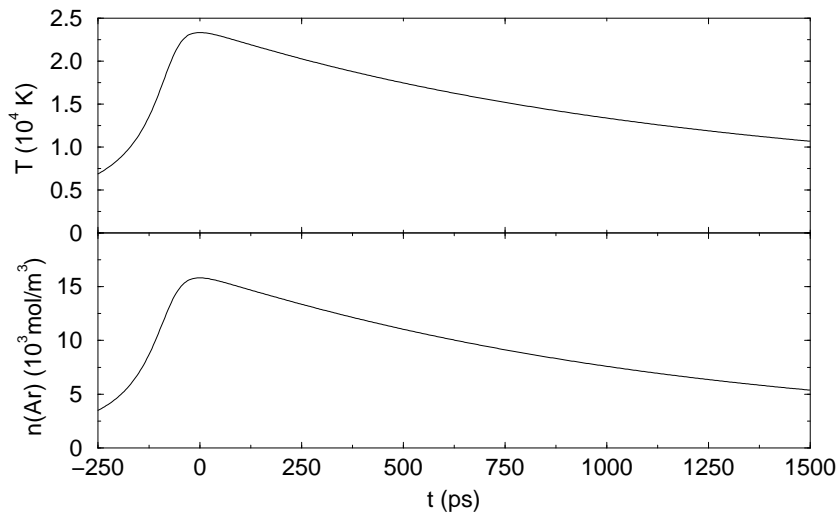

FIG. 5: Time profiles of temperature $T$ (upper panel) and number density of argon atom $n_{0}$ (lower panel) near the instant of minimum bubble size.

\section{Plasma and wave effects}

As mentioned previously, we employed two plasma collision models P1 and P2 in the simulations. The differences in these two models originate from the free-free (or free-bound) Gaunt factor and the Cillie exponential cut-off factor $\exp \left(-h f / k_{B} T\right)$. The exponential factor is usually close to unity in the Rayleigh-Jeans limit where $h f \ll k_{B} T$, but for the case of SL, $k_{B} T$ is of the order of a few $\mathrm{eV}$ and is within the range $1.5-6.2 \mathrm{eV}$ of the observed light spectrum, and hence is not negligible. In particular we find, when the bubble is at minimum size and the temperature and density of its contents are also at their maxima, the free-free Gaunt factor and the free-bound Gaunt factors, multiplied by the exponential cut-off, result in a correction factor of order 0.1 (see Fig. 6). Fig. 17 shows the computed power spectra obtained from simulations constructed respectively with $\mathrm{GOM} / \mathrm{WOM}+\mathrm{P} 1 / \mathrm{P} 2$, clearly demonstrating that the P2 model indeed leads to an decrease in the radiance.

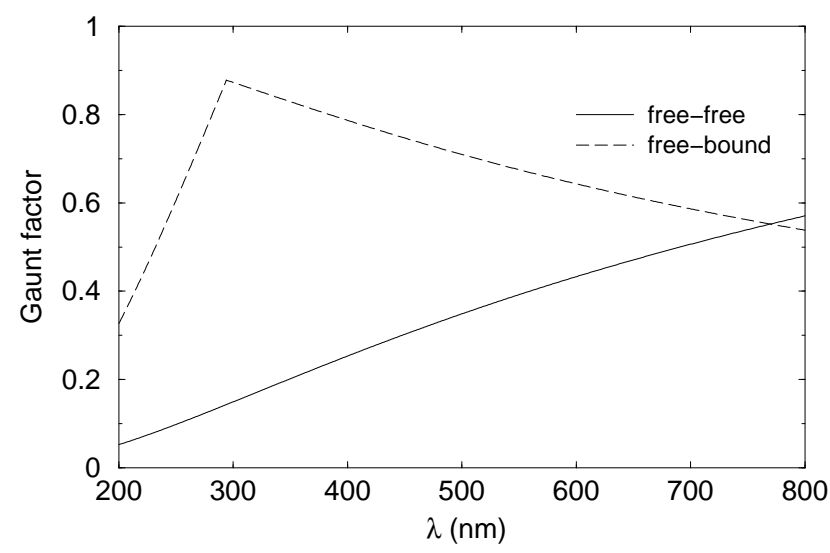

FIG. 6: Free-free (solid line) and free-bound (dashed line) Gaunt factors multiplied by the Cillie exponential factor at the instant of minimum bubble size.

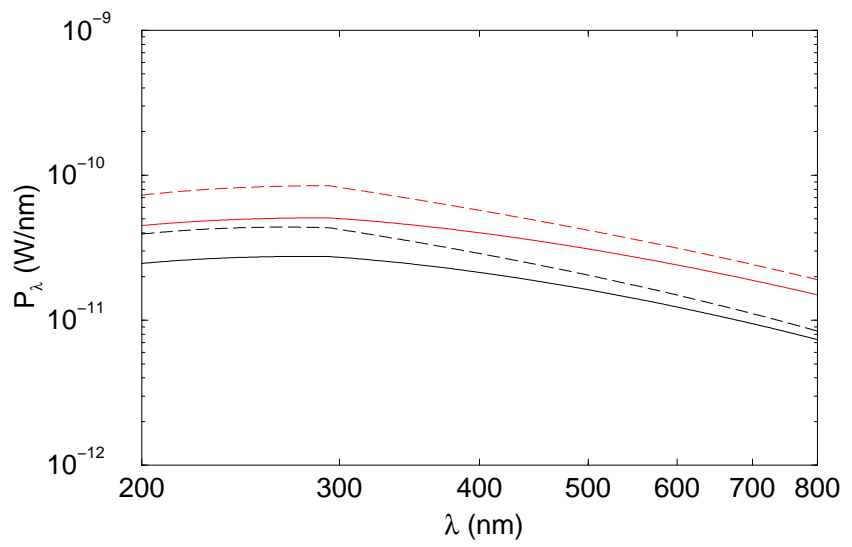

FIG. 7: (Color online) The spectral radiance $P_{\lambda}$ is plotted against the wavelength $\lambda$ for the following models: GOM $+\mathrm{P} 1$ (grey-dashed line), GOM+P2 (grey-solid line), $\mathrm{WOM}+\mathrm{P} 1$ (dark-dashed line), and WOM+P2 (dark-solid line)

Furthermore, we can observe in Fig. 7 that the power is overestimated when GOM is used rather than WOM, which can be readily explained as follows. When a plane wave is incident on a WOM bubble, part of it is reflected or scattered from the boundaries and the remaining part is absorbed as heat. In light of Kirchhoff's law then, less absorption implies less emission. In contrast, reflection and diffraction are neglected in GOM, resulting in an overestimated absorption and hence emission. Therefore, to achieve realistic power calculations comparable with experimental results, the effects of both the wave nature of light and the Gaunt factor correction cannot be neglected, consistent with the point we made earlier.

In subsequent discussions we will employ two specific light emission models: the model proposed in Ref. 20] $(\mathrm{GOM}+\mathrm{P} 1)$ and our present model $(\mathrm{WOM}+\mathrm{P} 2)$, and it should be understood that all power computations employing GOM are done with P1 while those employing WOM are done with P2. 


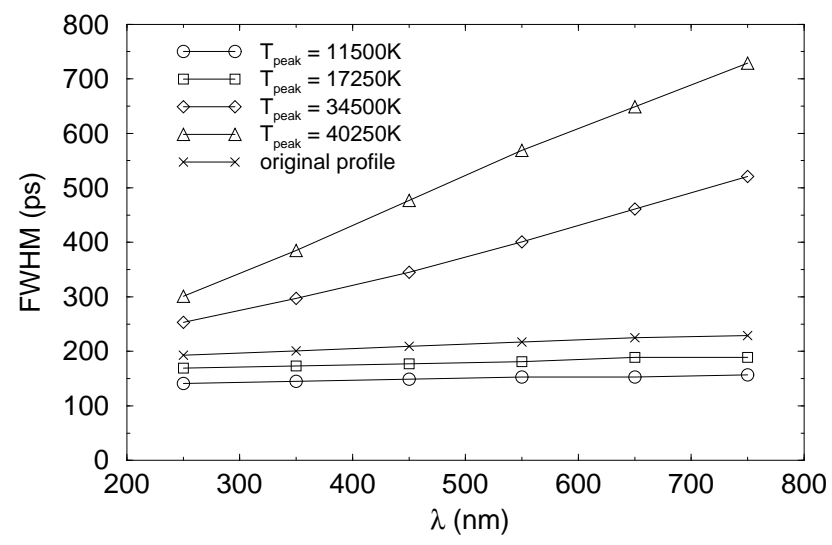

FIG. 8: FWHM obtained from UBM+GOM versus wavelength $\lambda$. Lines with circles, squares, crosses, diamonds and triangles respectively represent the cases with a scaling of 0.5 ; $0.75 ; 1$ (i.e. no scaling); 1.50 ; and 1.75 .

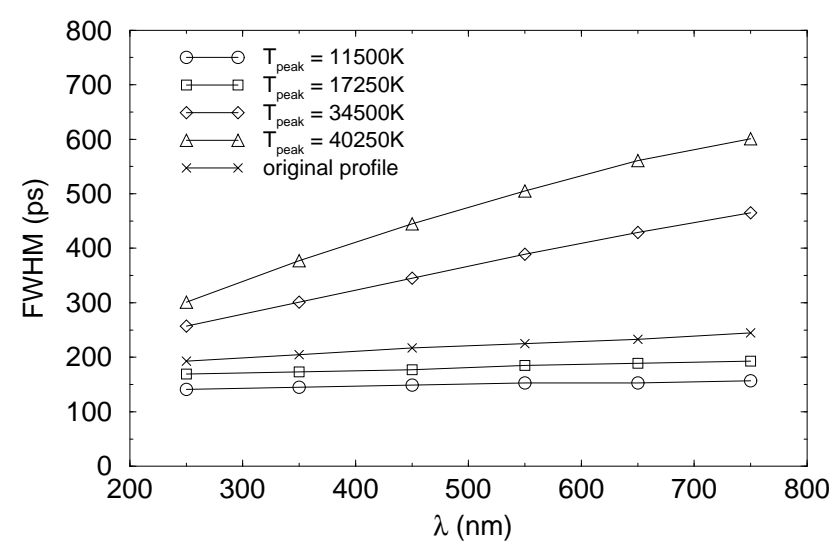

FIG. 9: FWHM obtained from UBM+WOM versus wavelength $\lambda$. Lines with circles, squares, crosses, diamonds and triangles respectively represent the cases with a scaling of 0.5 ; 0.75 ; 1 (i.e. no scaling); 1.50 ; and 1.75 .

\section{Temperature effects}

For our case studied here using the UBM model, the maximum temperature achieved at the instant of minimum bubble size is about $23000 \mathrm{~K}$ (Fig. 5). To study the effect of the interior temperature on light emission, we scale by hand the original temperature profile for $P_{a}=1.300 \mathrm{~atm}$ and $R_{0}=5.0 \mu \mathrm{m}$ by some chosen factors, say $0.3,0.5,0.75,1.5$ and 1.75 , while keeping the densities of Ar neutrals constant. Accordingly, two of the three input parameters to the light emission model (temperature and ion number density) are changed and one (the atom number density) remains fixed. We employ both GOM and WOM to calculate the power, and study the spectral variation of the FWHM calculated within $100 \mathrm{~nm}$ wavelength windows as shown in Figs. 8] and 9]

It is remarkable that a consistently smaller spectral variation of the FWHM is obtained when the light emission model WOM is used. The unscaled profile is about

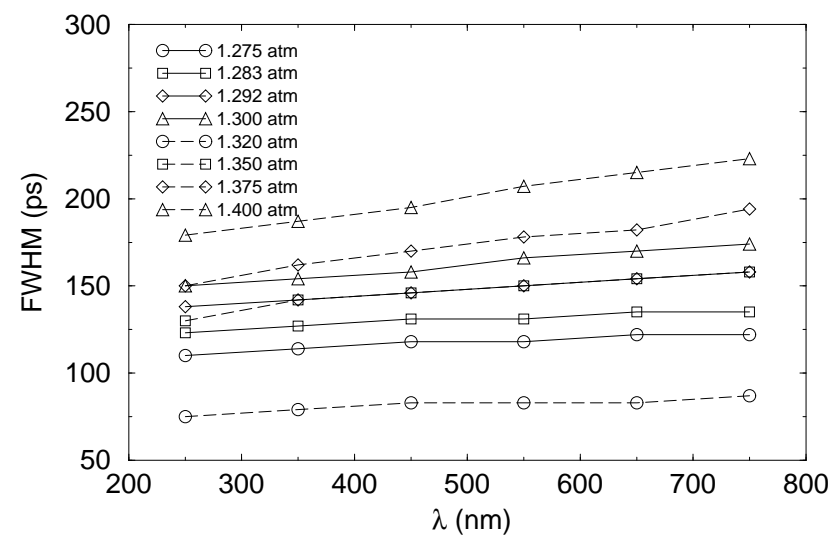

FIG. 10: FWHM versus wavelength $\lambda$ calculated from $\mathrm{UBM}+\mathrm{WOM}$. Cases with $T=20^{\circ} \mathrm{C}$ and $T=2.5^{\circ} \mathrm{C}$ are indicated by the solid curve and the dashed curve, respectively.

constant over the range of wavelength considered as in Ref. [20], which employed UBM and GOM, and the profile still remains remarkably constant when the temperature was scaled down by a factor of 0.5 and 0.75 (to a peak temperature of about $10000-20000 \mathrm{~K})$. Scaling down by 0.3 (to a peak temperature of about $7000 \mathrm{~K}$ ) produced zero power output since the temperature was much lower than that required for ionization. Nevertheless, scaling up by 1.5 and 1.75 (to a peak temperature of about $35000-45000 \mathrm{~K}$ ) produces dramatic variation of the FWHM. Hence, we find that the spectral uniformity of the FWHM holds only when the SL bubble temperature is restricted within a rather small range of moderate values. However, we remark that the temperature is underestimated in the UBM since local temperature rises were not taken account of. With a more realistic hydrodynamic modelling, Ref. 35] found the temperature should be several $10^{4} \mathrm{~K}$ higher. Thus, we expect that a larger FWHM spectral variation with increased driving pressure will be an essential realistic feature of SL.

In addition, we have studied the effect of ambient water temperature on SL light emission, using WOM and the values of $\left(P_{a}, R_{0}\right)$ extracted from the phase diagram in Ref. [52], at $T_{0}=20^{\circ} \mathrm{C}$ and $2.5^{\circ} \mathrm{C}$ and at a driving frequency of $f=26.5 \mathrm{kHz}$. Here we observe from Fig. 10

\begin{tabular}{|c|c|c|c|c|}
\hline \multicolumn{5}{|c|}{$T_{0}=20^{\circ} \mathrm{C}$} \\
\hline$P_{a}(\mathrm{~atm})$ & 1.275 & 1.283 & 1.292 & 1.300 \\
\hline$R_{0}(\mu \mathrm{m})$ & 2.9 & 3.2 & 3.6 & 3.9 \\
\hline \multicolumn{5}{|c|}{$T_{0}=2.5^{\circ} \mathrm{C}$} \\
\hline$P_{a}(\mathrm{~atm})$ & 1.320 & 1.350 & 1.375 & 1.400 \\
\hline$R_{0}(\mu \mathrm{m})$ & 2.0 & 3.5 & 4.0 & 4.5 \\
\hline
\end{tabular}

TABLE I: Driving pressure $P_{a}$ and ambient radius $R_{0}$ at $T_{0}=20^{\circ} \mathrm{C}$ and $T_{0}=2.5^{\circ} \mathrm{C}$ that give stable sonoluminescing bubble for $f=26.5 \mathrm{kHz}$.

the general trend of a larger FWHM towards the red end 
of the spectrum as the pressure is tuned up, and this increase is further enhanced at a lower water temperature. This is consistent with the experimental findings of Moran et al. [6] and is readily explained. At a lower water temperature, the bubble can be driven harder 52] so that, given a certain value of ambient radius, a larger driving pressure can be applied while maintaining bubble stability. The bubble collapses more violently under the larger pressure and hence the temperature of the bubble interior achieves a higher value, resulting in a larger FWHM spectral variation. Therefore, in effect, both of our observations under increased driving pressure and lower water temperature can be explained in terms of the higher temperature reached inside the bubble.

\section{B. Computational Fluid Mechanics Model}

\section{Hydrodynamics}

Now we employ CFM developed by Ho et al. 35. that includes the effects of ionizations and recombinations to calculate the power spectra and pulse profiles using WOM, and compare the results with those obtained from joint application of UBM and GOM. The set of conditions that we employ is extracted from Ref. 20 and shown in Table III where $T_{0}=20^{\circ} \mathrm{C}, f=20 \mathrm{kHz}$ and the dissolved gas concentration is $0.20 \%$. Figs. 11$]$ and 12 respectively show the computed results of UBM and CFM for a case with $P_{a}=1.325 \mathrm{~atm}, R_{0}=4.7 \mu \mathrm{m}$. It is found that the maximum temperature obtained with CFM can exceed $5 \times 10^{4} \mathrm{~K}$ while that in UBM is less than $3 \times 10^{4} \mathrm{~K}$. Accordingly, the number of $\mathrm{Ar}^{+}$ion in CFM is much greater than that in UBM.

\begin{tabular}{|c|c|c|c|c|}
\hline$P_{a}(\mathrm{~atm})$ & 1.275 & 1.300 & 1.325 & 1.350 \\
\hline$R_{0}(\mu \mathrm{m})$ & 2.6 & 4.0 & 4.7 & 5.4 \\
\hline
\end{tabular}

TABLE II: Driving pressure $P_{a}$ and ambient radius $R_{0}$ at $T_{0}=20^{\circ} \mathrm{C}$ that give stable sonoluminescing bubble for $f=$ $20 \mathrm{kHz}$.

\section{Light emission}

To apply WOM to CFM, which produces an inhomogeneous profile of bubble temperature and number densities, we approximate the resultant inhomogeneous profile by a layered one and use within each layer $j$ the average values of the temperature, the number densities of atom and electron there. The absorption coefficient $\kappa(j)$, collision frequency $\gamma(j)$ and refractive index $n(j)$ can accordingly be computed using these averaged values. This approximation scheme allows for the application of WOM to the resulting multilayered spherical configuration.
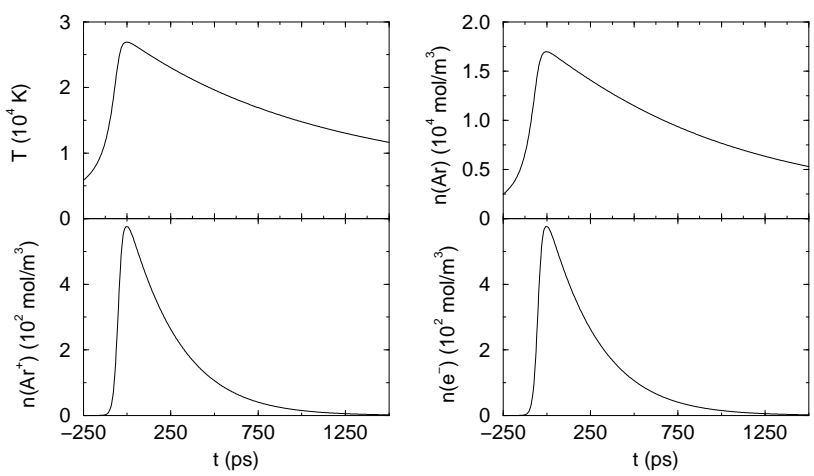

FIG. 11: Temperature $T$, number densities of argon neutrals $n(\mathrm{Ar})$, ions $n\left(\mathrm{Ar}^{+}\right)$and electrons $n\left(\mathrm{e}^{-}\right)$shown as a function of time, where $t=0$ is the instant of minimum bubble radius, for a UBM bubble with $P_{a}=1.325 \mathrm{~atm}$, and $R_{0}=4.7 \mu \mathrm{m}$.
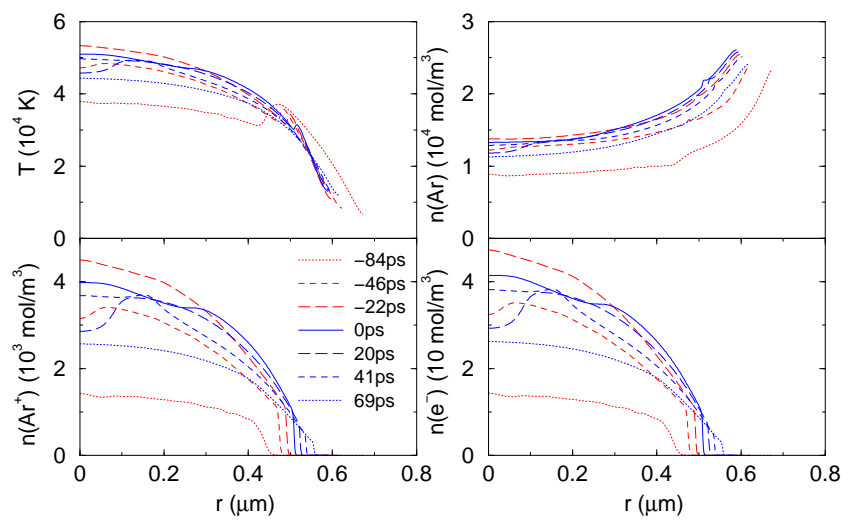

FIG. 12: (Color online) Snapshots near the instant of minimum bubble radius $(t=0)$ for the same quantities as in Fig. 11 plotted against radial distance, for a CFM bubble with $P_{a}=1.325 \mathrm{~atm}$, and $R_{0}=4.7 \mu \mathrm{m}$. Grey lines indicate times before zero while dark lines indicate times after zero.

As the degree of ionization in CFM result is much higher than that in UBM, the difference in the optical properties of these two models is obvious. As shown in Figs. 13] and 14] $\omega / \omega_{p}$ is reduced by a factor of 2.5 in CFM as compared to UBM. In particular, in CFM $\omega / \omega_{p}$ is close to unity near the UV end, indicating that plasma collective effects may be significant in the shortwavelength regime. Also, both dispersion and absorption are considerably stronger in the CFM case, exhibiting a larger variation in $n_{R}$ and a larger $n_{I}$ than the UBM case.

In Sec. VIB we have made the assumption that the plasma is so tenuous that the Coulomb energy is much smaller than the average thermal energy of individual particles and the plasma behaves like an ideal gas. The values of the ion-coupling parameter $\Gamma$ at the instant of maximum bubble compression for the cases studied here (see Table [II) are shown in Fig. 15] For UBM we note that $\Gamma \simeq 0.7$ for all four cases. In the CFM bubble, interestingly there is a clear formation of two regions: an 

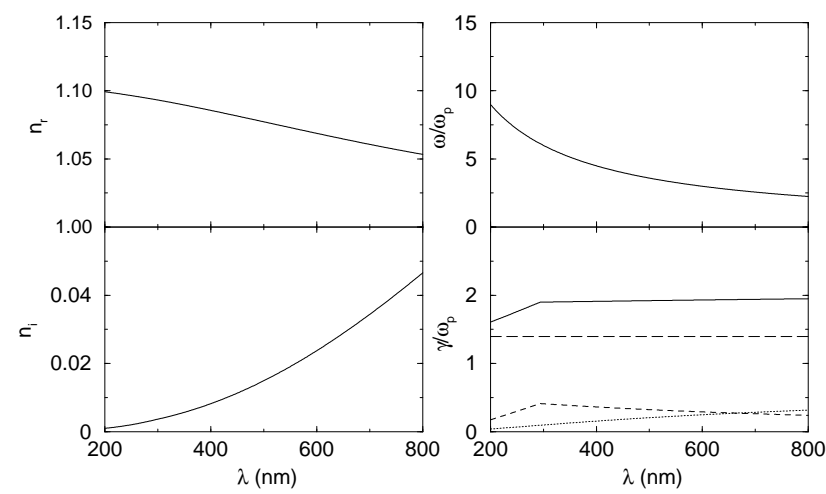

FIG. 13: Shown on the left are the real part $n_{R}$ and imaginary part $n_{I}$ of the refractive index, on the right are the ratios $\omega / \omega_{p}$ and $\nu / \omega_{p}$ versus the wavelength $\lambda$ for a UBM bubble with $P_{a}=1.375 \mathrm{~atm}$ and $R_{0}=2.6 \mu \mathrm{m}$ at the instant of minimum radius. In the graph $\nu / \omega_{p}$ versus $\lambda$, the dotted, short-dashed, long-dashed and full lines respectively show $\nu_{e i}, \nu_{r c}, \nu_{e a}$ and $\nu$.
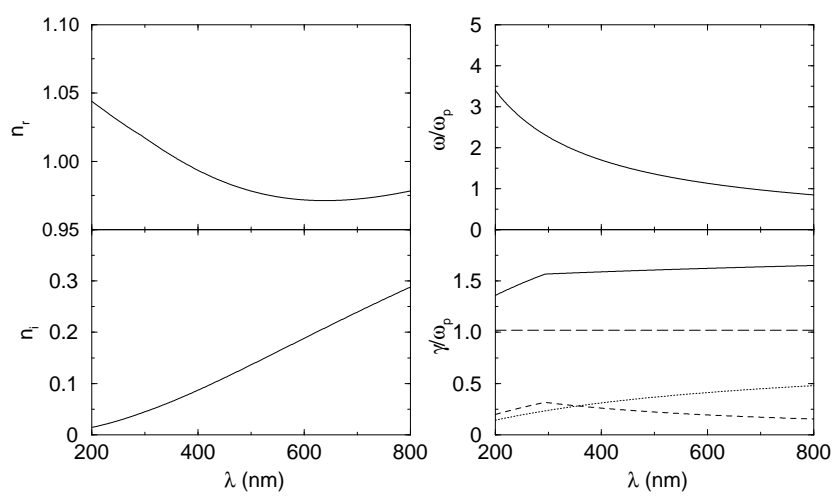

FIG. 14: Same as Fig. 13 for a CFM bubble with $P_{a}=$ $1.325 \mathrm{~atm}$, and $R_{0}=4.7 \mu \mathrm{m}$. The quantities shown here are those of the innermost layer which occupies the inner $7.5 \%$ of the bubble radius for $P_{a}=1.325 \mathrm{~atm}$ hence is representative of the hottest and densest region of the bubble.

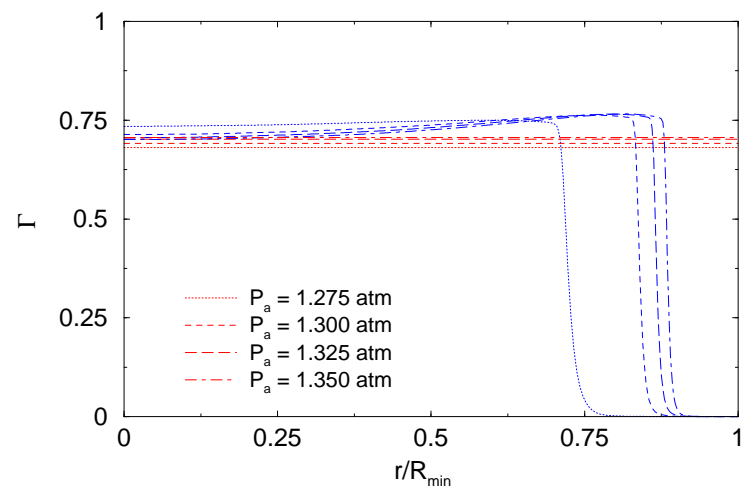

FIG. 15: (Color online) The ion-coupling parameter $\Gamma$ is plotted against $r$, normalized by the minimum radius $R_{\min }$ at the instant of maximal bubble compression. The values obtained from UBM (CFM) are indicated by grey (dark) lines.

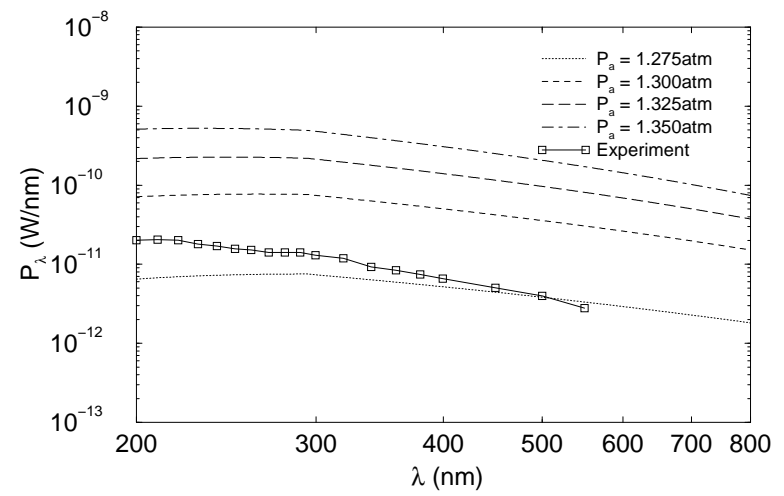

FIG. 16: Spectral radiance $P_{\lambda}$ versus wavelength $\lambda$ obtained from $\mathrm{GOM}+\mathrm{UBM}+\mathrm{P} 1$. The experimental spectrum is obtained from Barber et al. [1]

inner core which is moderately coupled $\Gamma \sim 0.7$ and an outer shell which is weakly coupled $\Gamma \simeq 0$. In addition the effect of increasing the driving pressure is seen to increase the size of this moderately-coupled inner core. Since the degree of coupling is moderate and not too strong, we expect that the formulas used for the absorption coefficients and collision frequencies based on the tenuous plasma assumption should still apply. However, on the other hand, if the driving pressure increases while maintaining the stability of oscillation, it is likely that the plasma might become a non-ideal one. The physical property of such dense non-ideal plasma is rather complicated and is beyond the scope of the present paper 47.

In the following we contrast data obtained respectively from $\mathrm{GOM}+\mathrm{UBM}$ and $\mathrm{WOM}+\mathrm{CFM}$ and specifically consider three different physical quantities, namely the spectrum, the pulse shape and the FWHM of light pulses.

In Figs. [16] and 17] we show the computed spectra using $\mathrm{GOM}+\mathrm{UBM}+\mathrm{P} 1$ and $\mathrm{WOM}+\mathrm{CFM}+\mathrm{P} 2$, respectively. One clearly sees the improvement (Fig. 17) of our refined model, namely $\mathrm{WOM}+\mathrm{CFM}+\mathrm{P} 2$, that the calculated spectral shape is much closer to the experimental results [1] than the GOM+UBM+P1 model. In particular, major improvement is seen in the UV portion of the spectrum.

The calculated pulse shapes are shown in Figs. 18 and 19 respectively, evidently the pulse shapes produced from $\mathrm{WOM}+\mathrm{CFM}+\mathrm{P} 2$ are more consistent with experimental data [4, 6, 53] where the long-time tail was not observed. By contrast, as shown Fig. [18 a long-time tail appears in $\mathrm{GOM}+\mathrm{UBM}+\mathrm{P} 1$ and is an undesirable feature.

Fig. 20] shows the calculated FWHM plotted against the wavelength for both cases. While it is clear that the FWHM increases with driving pressure as found in experiments [4, 6, 53], we also see that the FWHM remains nearly a constant over $200-800 \mathrm{~nm}$ only at a low pressure $P_{a} \sim 1.275 \mathrm{~atm}$ even in the more realistic $\mathrm{WOM}+\mathrm{CFM}+\mathrm{P} 2$ model (c.f. Sec. VIIIA 2). We remark that the results obtained by Gompf et al. [4] showing sim- 


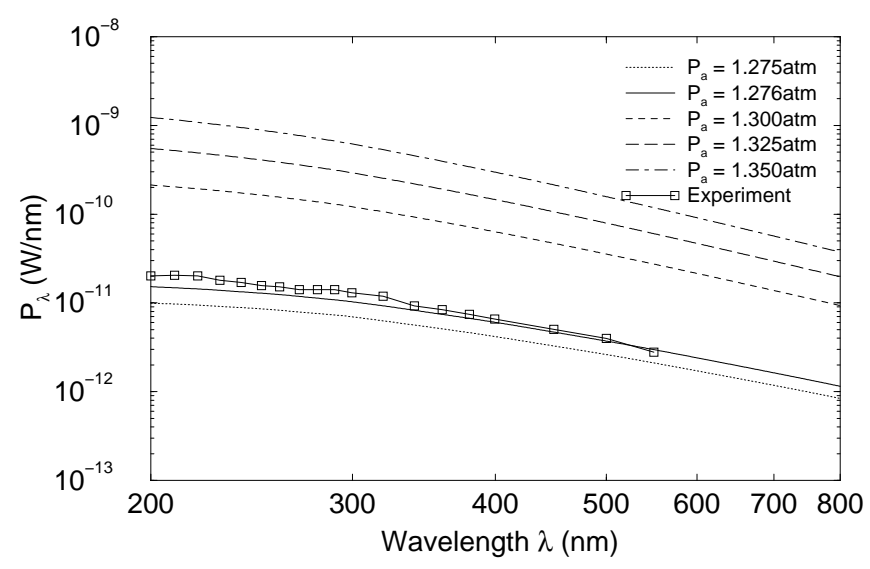

FIG. 17: Spectral radiance $P_{\lambda}$ versus wavelength $\lambda$ obtained from $\mathrm{WOM}+\mathrm{CFM}+\mathrm{P} 2$. The best-fit for the experimental spectrum [1] is indicated by the solid line, in which $P_{a}=1.276 \mathrm{~atm}, R_{0}=2.7 \mu \mathrm{m}$.

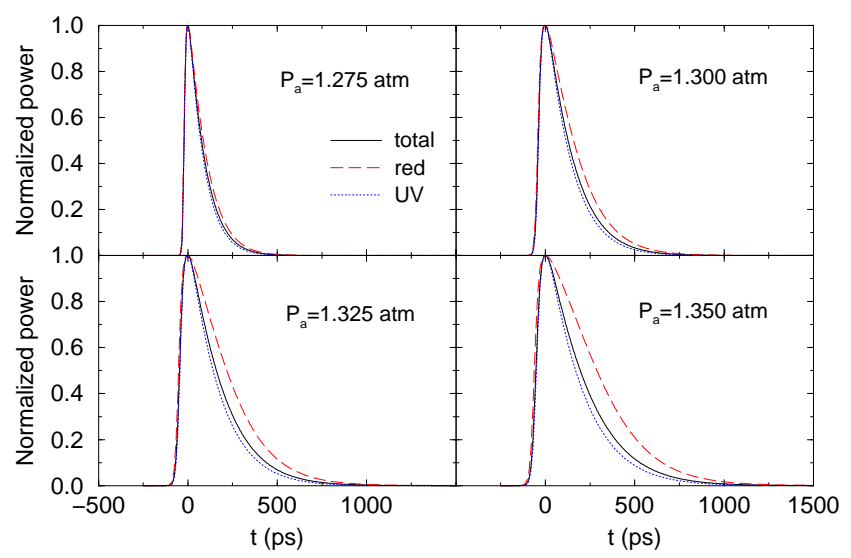

FIG. 18: (Color online) Normalized power versus time obtained from GOM+UBM+P1. The solid, dashed, and dotted lines are respectively the total normalized power, the normalized powers in the red and UV regions.

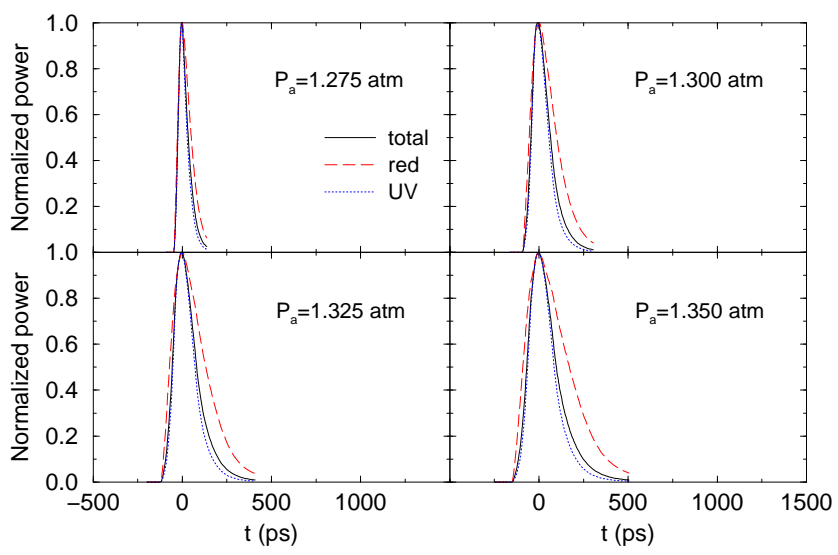

FIG. 19: (Color online) Normalized power versus time obtained from $\mathrm{WOM}+\mathrm{CFM}+\mathrm{P} 2$. The solid, dashed, and dotted lines are respectively the total normalized power, the normalized powers in the red and UV regions.

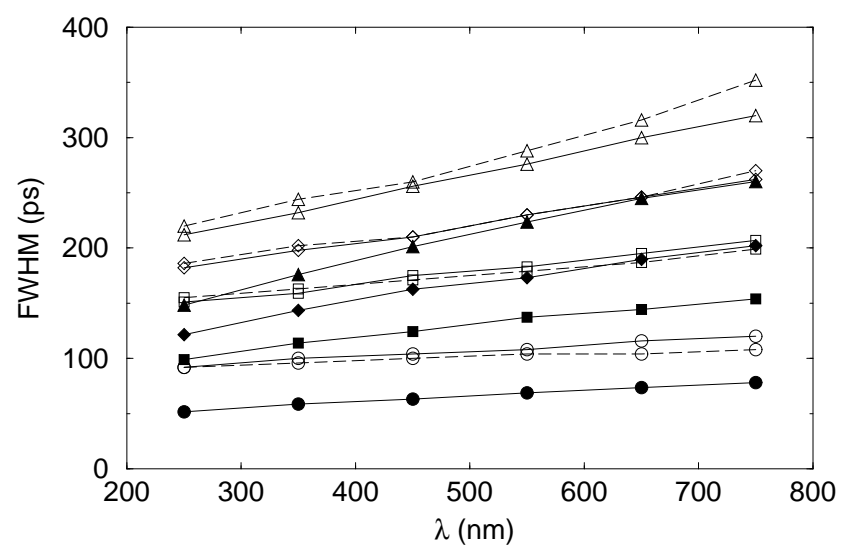

FIG. 20: FWHM of light pulse versus wavelength $\lambda$ obtained at different driving pressures: circle $P_{a}=1.275 \mathrm{~atm}$, square $P_{a}=1.300 \mathrm{~atm}$, diamond $P_{a}=1.325 \mathrm{~atm}$ and triangle $P_{a}=$ $1.350 \mathrm{~atm}$. Dashed lines with empty symbols and solid lines with filled symbols are calculated from $\mathrm{GOM}+\mathrm{UBM}+\mathrm{P} 1$ and $\mathrm{WOM}+\mathrm{CFM}+\mathrm{P} 2$, respectively.

ilar pulse widths for the red and UV pulse were obtained under a driving pressure of $P_{a}=1.200 \mathrm{~atm}$; which is smaller than the lowest pressure $P_{a}=1.275 \mathrm{~atm}$ we used and is therefore expected to show a constant pulse width. In particular the results of Moran et al. [6] showed the FWHM increases with wavelength at a low ambient water temperature $3{ }^{\circ} \mathrm{C}$, which, as remarked previously, also resulted in a larger driving pressure. Thus we emphasize that in general the notion of wavelength-independence of the SL pulse width is only correct at low driving pressures; and at higher driving pressures spectral dispersion of the pulse width shows up, and this can be simply explained within our model. Either effects of higher driving pressure or lower water temperature boil down to the consequence of higher bubble temperature. As the bubble becomes hotter, both optical dispersion and absorption become more significant and hence the bubble becomes more optically opaque, approaching a blackbody surface emitter. Red light is then radiated for a longer duration than the UV since, throughout one cycle, the bubble can stay at a lower-temperature state for a longer duration. As a consequence, the pulse width increases towards the red end of the spectrum. In other words, the emitted light becomes more spectrally dispersive because of the increased absorption and dispersion in the plasma medium. In particular, the absorption is highest (Figs.13 and 14) at the red end of the spectrum, resulting in a flatter pulse shape there (larger FWHM). Thus, besides using a lower ambient temperature as in Ref. [6], if a strong enough pressure is applied (while still maintaining bubble stability) the spectral variation of the FWHM would be an observable consequence in experiment. 


\section{CONCLUSION}

In summary, we have proposed in the present paper a robust theory for optical emission in SBSL that properly takes into account of the wave nature and propagation of light in the absorptive plasma formed inside a sonoluminescing bubble in a self-consistent way. In addition, our theory can be applied to bubbles with inhomogeneous density and temperature profiles. The validity of our scheme was examined for the case of SBSL with argon bubbles. By introducing and implementing appropriate Gaunt factors and exponential correction in the collision frequencies; as well as the effects of optical thickness, scattering, reflection and diffraction, our light emission model successfully explains the major features (including power spectrum, pulse shape and FWHM) observed in SBSL experiments. In addition, the computed power spectra and pulse shapes are shown to be in excellent agreement with experimental results.

Besides, we have also shown that the experimentally observed spectral independence of the FWHM at $T_{0}=$ $20^{\circ} \mathrm{C}$ is ascribable to the relatively small temperature range (about $10000-30000 \mathrm{~K}$ ) achievable in a collapsing SL bubble. Above this range the bubble behaves in the way of a blackbody surface emitter and the spectral variation of the FWHM should be more notable. In fact, as the driving pressure goes up, the temperature reached inside the bubble rises. Also, if the ambient water temperature is lowered at a fixed ambient radius $R_{0}$, the driving pressure allowable for bubble stability extends to a larger value. This provides a theoretical explanation for why Moran et al. [6] found a spectral variation of the pulse width at $T=3{ }^{\circ} \mathrm{C}$.

Notwithstanding the above-mentioned achievements, the model developed in the present paper is only one of the many steps towards a better understanding of SBSL, which is an extremely complex phenomenon resulting from the subtle interplay of hydrodynamics, chemical reactions, plasma physics and optics as well. Much more challenging problems, e.g. SBSL with inert gases other than argon and inclusion of water vapor in the hydrodynamic code, are still ahead for us. They are surely our goal of endeavor in the future.

\section{Acknowledgments}

We thank M.-C. Chu and K.M. Pang for discussions. The work described in this paper was partially supported by a grant (Project No. 401603) from the Research Grants Council of the Hong Kong Special Administrative Region, China.
[1] B. P. Barber, R. A. Hiller, R. Löfstedt, S. J. Putterman, and K. R. Weninger, Phys. Rep. 281, 65 (1997).

[2] M. P. Brenner, S. Hilgenfeldt, and D. Lohse, Rev. Mod. Phys. 74, 425 (2002).

[3] B. P. Barber and S. J. Putterman, Nature 352, 318 (1991).

[4] B. Gompf, R. Gunther, G. Nick, R. Pecha, and W. Eisenmenger, Phys. Rev. Lett. 79, 1405 (1997).

[5] R.A. Hiller, S. J. Putterman, and K.R. Weninger, Phys. Rev. Lett. 80, 1090 (1998).

[6] M. J. Moran and D. Sweider, Phys. Rev. Lett. 80, 4987 (1998).

[7] R. Hiller, S. J. Putterman, and B. P. Barber, Phys. Rev. Lett. 69, 1182 (1992).

[8] R. Hiller, K. Weninger, S. J. Putterman, and B. P. Barber, Science 266, 248 (1994).

[9] D. F. Gaitan, L. A. Crum, C. C. Church, and R. A. Roy, J. Acoust. Soc. Am. 91, 3166 (1992).

[10] R. Löfstedt, B. B. Barber, and S. J. Putterman, Phys. Fluids A 5, 2911 (1993).

[11] R. Löfstedt, K. Weninger, S. Putterman, and B. P. Barber, Phys. Rev. E 51, 4400 (1995).

[12] C. C. Wu and P. H. Roberts, Phys. Rev. Lett. 70, 3424 (1993).

[13] W. C. Moss, D. B. Clarke, and D. A. Young, Science 276, 1398 (1997).

[14] L. Yuan, H. Y. Cheng, M.-C. Chu, and P. T. Leung, Phys. Rev. E 57, 4265 (1998).

[15] H. Y. Cheng, M.-C. Chu, P. T. Leung, and L. Yuan, Phys. Rev. E 58, R2705 (1998).

[16] W. C. Moss et al., Phys. Rev. E 59, 2986 (1999).
[17] G. Vazquez, C. Camara, S. J. Putterman, and K. Weninger, Phys. Rev. Lett. 88, 197402 (2002).

[18] G. Vazquez, C. Camara, S. J. Putterman, and K. Weninger, Opt. Lett. 26, 575 (2001).

[19] N. Xu, L. Wang, and X. Hu, Phys. Rev. E 57, 1615 (1998).

[20] S. Hilgenfeldt, S. Grossmann, and D. Lohse, Phys. Fluids 11, 1318 (1999).

[21] S. Hilgenfeldt, S. Grossmann, and D. Lohse, Nature 398, 402 (1999).

[22] L. Frommhold and A. A. Atchley, Phys. Rev. Lett. 73, 2883 (1994).

[23] L. Frommhold, Phys. Rev. E 58, 1899 (1998).

[24] C. Eberlein, Phys. Rev. Lett. 76, 3842 (1996).

[25] L. S. Bernstein and M. R. Zakin, J. Phys. Chem. 99, 14619 (1995)

[26] J. R. Willison, Phys. Rev. Lett. 81, 5430 (1998).

[27] B. P. Barber, C. C. Wu, R. Löfstedt, P. H. Roberts, and S. J. Putterman, Phys. Rev. Lett. 72, 1380 (1994).

[28] R. P. Taleyarkhan, C. D. West, J. S. Cho, J. R. T. Lahey, R. I. Nigmatulin, and R. C. Block, Science 295, 1868 (2002).

[29] R. P. Taleyarkhan, J. S. Cho, C. D. West, J. R. T. Lahey, R. I. Nigmatulin, and R. C. Block, Phys. Rev. E 69, 036109 (2004).

[30] S. J. Putterman, P. G. Evans, G. Vazquez, and K. Weninger, Nature 409, 782 (2001).

[31] C. Camara, S. Putterman, and E. Kirilov, Phys. Rev. Lett. 92, 124301 (2005).

[32] S. D. Hopkins, S. J. Putterman, B. A. Kappus, K. S. Suslick, and C. G. Camara, Phys. Rev. Lett. 95, 254301 
(2005).

[33] D. J. Flannigan and K. S. Suslick, Nature 434, 52 (2005).

[34] S. M. Rytov, Y. A. Kravtsov, and V. I. Tatarskii, Principles of statistical radiophysics 3: Elements of Random Fields (Springer-Verlag, Berlin, 1989).

[35] C. Y. Ho, L. Yuan, M.-C. Chu, P. T. Leung, and W. Wei, Phys. Rev. E 65, 041201 (2002).

[36] T. W. Chen, P. T. Leung, and M.-C. Chu, Phys. Rev. E 62, 6584 (2000).

[37] G. Bekefi, Radiation processes in plasmas (Wiley, New York, 1966).

[38] D. J. Landau and E. M. Lifshitz, Electrodynamics of Continuous Media (Pergamon, Oxford, 1960).

[39] Y. B. Zel'dovich and Y. P. Raizer, Physics of Shock Waves and High-Temperature Hydrodynamic Phenomena, Vol. I and II (Academic Press, New York and London, 1966).

[40] D. A. Varshalovich, A. N. Moskalev, and V. K. Khersonskii, Quantum theory of angular momentum: irreducible tensors, spherical harmonics, vector coupling coefficients, 3nj symbols (World Scientific, Singapore, 1988).

[41] J. D. Jackson, Classical Electrodynamics (Wiley, New York, 1999), 3rd ed.

[42] S. C. Brown, Basic data of plasma physics (MIT Press and Wiley, New York, 1959).

[43] M. A. Heald and C. B. Wharton, Plasma diagnostics with microwaves (Wiley, New York, 1965).

[44] P. J. Brussaard and H. C. V. D. Hulst, Rev. Mod. Phys. 34, 507 (1962).

[45] S. Nozawa, N. Itoh, and Y. Kohyama, Astrophy. J. 507, 530 (1998).

[46] N. Itoh, S. Kusano, T. Sakamoto, S. Nozawa, and Y. Kohyama, Astrophy. J. Supp. 128, 125 (2000).

[47] V. Fortov and I. Iakubov, The Physics of Non-Ideal Plasma (World Scientific, Singapore, 2000).

[48] V. Kamath and A. Prosperetti, J. Acoust. Soc. Am. 85, 1538 (1987).

[49] A. Prosperetti and A. Lezzi, J. Fluid Mech. 168, 457 (1986).

[50] N. Xu, L. Wang, and X. Hu, Phys. Rev. Lett. 83, 2441 (1999).

[51] A. Prosperetti, J. Acoust. Soc. Am. 61, 17 (1977).

[52] S. Hilgenfeldt, D. Lohse, and W. C. Moss, Phys. Rev. Lett. 80, 1332 (1998).

[53] R. Pecha, B. Gompf, G. Nick, Z. Q. Wang, and W. Eisenmenger, Phys. Rev. Lett. 81, 717 (1998). 